\title{
Existence Theorems for Vortices in the Aharony-Bergman-Jaferis-Maldacena Model
}

\author{
Xiaosen Han \\ Institute of Contemporary Mathematics \\ School of Mathematics \\ Henan University \\ Kaifeng, Henan 475004, PR China \\ Yisong Yang \\ Department of Mathematics \\ Polytechnic Institute of New York University \\ Brooklyn, New York 11201, USA
}

\begin{abstract}
A series of sharp existence and uniqueness theorems are established for the multiple vortex solutions in the supersymmetric Chern-Simons-Higgs theory formalism of Aharony, Bergman, Jaferis, and Maldacena, for which the Higgs bosons and Dirac fermions lie in the bifundamental representation of the general gauge symmetry group $U(N) \times U(N)$. The governing equations are of the BPS type and derived by Kim, Kim, Kwon, and Nakajima in the mass-deformed framework labeled by a continuous parameter.
\end{abstract}

\section{Introduction}

It is well known that the presence of the Chern-Simons terms in field theories is essential in many areas of modern physics, especially for condensed matter systems [20 22, 50]. More recently, the importance of the Chern-Simons terms in superstring theory and M-theory was explored in a general formalism by Schwarz [41], in the context of some Lagrangian descriptions of superconformal gauge field theories which couple the Chern-Simons gauge and matter fields. This formalism was then made into fruition by Bagger and Lambert [5] 7] and Gustavsson [25], which has since commonly been referred to as the Bagger-Lambert-Gustavsson (BLG) theory [9, 13, 18]. Shortly afterwards, Aharony, Bergman, Jafferis, and Maldacena (ABJM) developed an elegant bi-level Chern-SimonsHiggs theory [2], extending the BLG theory. In both the BLG and ABJM theories, topological solitons realized as the classical solutions of various BPS (named after the pioneering works of Bogomol'nyi [10] and Prasad-Sommerfield [40]) equations known as fuzzy funnels, domain walls, and vortices, are basic field configurations that describe M-branes. In the present study, we aim at establishing an existence and uniqueness theory for the multiple vortex solutions in a general class of BPS equations in the ABJM model. It is interesting to note that, in these superconformal 
Lagrangian field theories [2,5 7, 41], the dynamics of the gauge fields is exclusively of the ChernSimons type, meaning that the usual quadratic kinetic terms involving the gauge field strength tensors, are missing [41], as in the earlier Chern-Simons-Higgs models of Hong-Kim-Pac [28] and Jackiw-Weinberg [30]. In these latter studies [28,30] and the subsequent development of the subject (cf. [16] and references therein), suitable six-order Higgs potential density functions have to be taken in order to achieve a BPS reduction. While, in such a situation, the BPS reductions can be made, the six-order potential terms lead to several complications or drawbacks of the models. The first one is that it is not clear whether topological solutions are uniquely determined by any prescribed distribution of vortices, as seen in the classical Abelian Higgs theory [31,47], although the existence of maximal solutions has been established [43]. The second one is the occurrence of non-topological solutions [29,44] which are plagued by non-uniqueness and the issue of existence of solutions realizing arbitrarily prescribed vortices has only been tackled in some extreme cases [12, 14]. The third one concerns the solutions over a doubly-periodic domain resembling a vortex condensate/lattice structure [1,11,48] and it is known that even in such a compact setting non-uniqueness occurs [45] and the interval ensuring the existence of a solution for the Chern-Simons coupling constant cannot be explicitly determined [11,45]. Furthermore, for non-Abelian BPS vortex equations, although the existence of topological solutions can be proved [51], the existence of vortex condensates has only been established for rank 2 gauge groups [39] and the existence of non-topological solutions is still unsettled [46, 53]. For the Chern-Simons vortices arising in the ABJM theory, however, we shall see through the present work that the situation is totally different. More precisely, we will develop a complete existence theory for multiple vortex solutions arising in the ABJM theory with the general gauge group $U(N) \times U(N)$ which gives us a unique topological solution for any prescribed distribution of vortices in the plane and a unique doubly periodic condensate solution under explicitly stated necessary and sufficient conditions involving several related physical coupling parameters. Furthermore, the model will be seen to be free of non-topological solutions.

The governing BPS equations considered here for the multiple vortex solutions in the ABJM theory were discovered in the work of Kim-Kim-Kwon-Nakajima [33] who showed that for the bottom case when the gauge group is $U(2) \times U(2)$ the equations can be reduced to that of the Abelian Higgs equation [24, 31, 38] which has been thoroughly understood [31, 47] and that for the general case with the gauge group $U(N) \times U(N)$ (with $N \geq 3$ ) the equations are given as an $(N-1$ ) by $(N-1)$ system of nonlinear elliptic equations of delicate structures. These equations arise in the so-called 'mass-deformed' theory labeled by a mass deformation parameter $\mu \geq 0$. When $\mu=0$ (mass deformation is absent), it has been shown in [33] that there is no finite-energy regular solution, and that finite-energy solutions can only be expected to appear when $\mu>0$ (mass deformation is present). The aim of our work here is to establish an existence theory for such mass-deformed ABJM vortex equations. A similar structure has also been seen in the independent work of Auzzi and Kumar [4] for which some existence and uniqueness theorems have been obtained in [35] through exploiting appropriate variational methods. Enlightened by the success of that study, our technical strategy in the current study will again be to explore and unveil the underlying variational structures of the system. It is interesting to notice that, although the Higgs potential density function still contains six-order terms, which play a crucial role for generating non-topological vortices in the classical Chern-Simons-Higgs models [29,44], such vortices are absent in the ABJM situation.

The content of the rest of the paper is outlined as follows. In Section 2, we recall the multiple 
vortex equations derived in the work of Kim-Kim-Kwon-Nakajima [33] in the ABJM model [2] and state our main existence results. The subsequent sections are then devoted to proofs of results. Specifically, in Section 3, we first apply a matrix decomposition procedure to unveil the variational structure of the system of governing elliptic equations. We next prove the existence of a solution by a somewhat indirect minimization approach, using the method of [31,47]. We then obtain the asymptotic decay properties of the solution on the full plane and calculate the anticipated quantized integrals which give rise to quantized vortex fluxes. In Section 4, we consider the compact case when solutions are doubly periodic. We shall mainly adapt the direct minimization method used in [35]. In Section 5, we present the limiting case $a=0$ which is of independent interest. We will see that such a limiting case allows us to state our necessary and sufficient conditions for the existence of a doubly periodic multiple solution explicitly. In Section 6 , we reconsider the case when $a>0$ and present two concrete examples, with $N=3$ and $N=4$, respectively, as an illustration of the application of our results in the general situation $(a>0)$. We shall also describe how to use our results to estimate the dimension of the moduli space of the BPS equations in the ABJM model under consideration.

\section{Existence of vortices in the ABJM model}

Use $\mu, \nu=0,1,2$ to denote the Lorentzian indices of the Minkowski spacetime $\mathbb{R}^{2,1}$ of signature $(-++)$. Like the BLG model [5], 2, 25], the ABJM model [2, 8] is formulated as a low-energy approximation of multiple M2-branes so that it is dual to M-theory on appropriate anti-de Sitter orbifolds. It is an $\mathcal{N}=6$ supersymmetric Chern-Simons theory with the gauge group $U(N) \times U(N)$ governed by the full Lagrangian density

$$
\mathcal{L}_{\mathrm{ABJM}}=\mathcal{L}_{\mathrm{CS}}+\mathcal{L}_{\text {kin }}-V_{\text {ferm }}-V_{0},
$$

in which the Chern-Simons Lagrangian $\mathcal{L}_{\mathrm{CS}}$ is given by

$$
\mathcal{L}_{\mathrm{CS}}=\frac{k}{4 \pi} \epsilon^{\mu \nu \lambda} \operatorname{Tr}\left(A_{\mu} \partial_{\nu} A_{\lambda}+\frac{2 \mathrm{i}}{3} A_{\mu} A_{\nu} A_{\lambda}-\hat{A}_{\mu} \partial_{\nu} \hat{A}_{\lambda}-\frac{2 \mathrm{i}}{3} \hat{A}_{\mu} \hat{A}_{\nu} \hat{A}_{\lambda}\right)
$$

which describes two Lie algebra $u(N)$-valued gauge fields, $A_{\mu}$ and $\hat{A}_{\mu}$, with the Chern-Simons level $(k,-k)$; using $\gamma^{\mu}$ to denote the Dirac matrices expressible in terms of the standard Pauli spin matrices, $\gamma^{0}=\mathrm{i} \sigma^{2}, \gamma^{1}=\sigma^{1}, \gamma^{2}=\sigma^{3}$, the matter-kinetic Lagrangian $\mathcal{L}_{\text {kin }}$ is given by

$$
\mathcal{L}_{\text {kin }}=-\operatorname{Tr}\left(D_{\mu} Y_{A}^{\dagger} D^{\mu} Y^{A}\right)+\mathrm{i} \operatorname{Tr}\left(\psi^{A \dagger} \gamma^{\mu} D_{\mu} \psi_{A}\right)
$$

which couple four complex scalars $Y_{A}(A=1,2,3,4)$ and four Dirac fermions $\psi_{A}(A=1,2,3,4)$ in

the bifundamental representations $(\mathbf{N}, \overline{\mathbf{N}})$ of the gauge group so that the gauge-covariant derivatives take the form

$$
\left.\begin{array}{rl}
D_{\mu} Y^{A} & =\partial_{\mu} Y^{A}+\mathrm{i} A_{\mu} Y^{A}-\mathrm{i} Y^{A} \hat{A}_{\mu}, \\
D_{\mu} Y_{A} & =\partial_{\mu} Y^{A}+\mathrm{i} \hat{A}_{\mu} Y_{A}-\mathrm{i} Y_{A} A_{\mu},
\end{array}\right\} \quad A=1,2,3,4
$$

the Yukawa-like quartic-interaction potential density $V_{\text {ferm }}$ is given by

$$
\begin{aligned}
V_{\text {ferm }}= & \frac{2 \pi \mathrm{i}}{k} \operatorname{Tr}\left(Y_{A}^{\dagger} Y^{A} \psi^{B \dagger} \psi_{B}-Y^{A} Y_{A}^{\dagger} \psi_{B} \psi^{B \dagger}+2 Y^{A} Y_{B}^{\dagger} \psi_{A} \psi^{B \dagger}\right. \\
& \left.-2 Y_{A}^{\dagger} Y^{B} \psi^{A \dagger} \psi_{B}-\epsilon^{A B C D} Y_{A}^{\dagger} \psi_{B} Y_{C}^{\dagger} \psi_{D}+\epsilon_{A B C D} Y^{A} \psi^{B \dagger} Y^{C} \psi^{D \dagger}\right)
\end{aligned}
$$


and $V_{0}$ is the sextic scalar potential

$$
\begin{aligned}
& V_{0}= \\
& \frac{4 \pi^{2}}{3 k^{2}} \operatorname{Tr}\left(6 Y^{A} Y_{B}^{\dagger} Y^{B} Y_{A}^{\dagger} Y^{C} Y_{C}^{\dagger}-Y^{A} Y_{A}^{\dagger} Y^{B} Y_{B}^{\dagger} Y^{C} Y_{C}^{\dagger}-Y_{A}^{\dagger} Y^{A} Y_{B}^{\dagger} Y^{B} Y_{C}^{\dagger} Y^{C}-4 Y^{A} Y_{B}^{\dagger} Y^{C} Y_{A}^{\dagger} Y^{B} Y_{C}^{\dagger}\right) .
\end{aligned}
$$

With the Lagrangian density given by (2.1), the action

$$
\mathcal{A}_{\mathrm{ABJM}}=\int \mathcal{L}_{\mathrm{ABJM}} \mathrm{d} x
$$

evaluated over the full spacetime is invariant under the $\mathcal{N}=6$ supersymmetry transformation [2,33]

$$
\begin{aligned}
\delta Y^{A} & =\mathrm{i} \omega^{A B} \psi_{B}, \\
\delta \psi_{A} & =-\gamma_{\mu} \omega_{A B} D_{\mu} Y^{B}+\frac{2 \pi}{k}\left(-\omega_{A B}\left[Y^{C} Y_{C}^{\dagger} Y^{B}-Y^{B} Y_{C}^{\dagger} Y^{C}\right]+2 \omega_{B C} Y^{B} Y_{A}^{\dagger} Y^{C}\right) \\
& \equiv-\gamma_{\mu} \omega_{A B} D_{\mu} Y^{B}+\omega_{B C}\left(\beta_{A}^{B C}+\delta_{A}^{[B} \beta_{D}^{C] D}\right), \\
\delta A_{\mu} & =-\frac{2 \pi}{k}\left(Y^{A} \psi^{B \dagger} \gamma_{\mu} \omega_{A B}+\omega^{A B} \gamma_{\mu} \psi_{A} Y_{B}^{\dagger}\right), \\
\delta \hat{A}_{\mu} & =\frac{2 \pi}{k}\left(\psi^{A \dagger} Y^{B} \gamma_{\mu} \omega_{A B}+\omega^{A B} \gamma_{\mu} Y_{A}^{\dagger} \psi_{B}\right),
\end{aligned}
$$

where $\omega_{A B}$ are supersymmetry transformation parameters satisfying

$$
\omega^{A B}=\left(\omega_{A B}\right)^{*}=-\frac{1}{2} \epsilon^{A B C D} \omega_{C D}
$$

and $\beta_{C}^{A B}$ are given by the expressions

$$
\beta_{C}^{A B}=\frac{4 \pi}{k} Y^{[A} Y_{C}^{\dagger} Y^{B]}=\frac{4 \pi}{k}\left(Y^{A} Y_{C}^{\dagger} Y^{B}-Y^{B} Y_{C}^{\dagger} Y^{A}\right)
$$

In the mass-deformed theory, we need to update the Lagrangian density (2.1) by modifying the potential densities $V_{\text {ferm }}$ and $V_{0}$ following the recipe

$$
V_{\text {ferm }} \mapsto V_{\text {ferm }}+\Delta V_{\text {ferm }}, \quad V_{0} \mapsto V_{0}+\Delta V_{0} \equiv V_{m},
$$

where

$$
\begin{aligned}
\Delta V_{\mathrm{ferm}} & =\operatorname{Tr}\left(\mu \psi^{\dagger A} M_{A}^{B} \psi_{B}\right), \\
\Delta V_{0} & =\operatorname{Tr}\left(\frac{4 \pi \mu}{k} Y^{A} Y_{A}^{\dagger} Y^{B} M_{B}^{C} Y_{C}^{\dagger}-\frac{4 \pi \mu}{k} Y_{A}^{\dagger} Y^{A} Y_{B}^{\dagger} M_{C}^{B} Y^{C}+\mu^{2} Y_{A}^{\dagger} Y^{A}\right),
\end{aligned}
$$

in which $\mu>0$ is a mass-deformation parameter which should not be confused with the Lorentzian index and $M_{A}^{B}=\operatorname{diag}\{1,1,-1,-1\}$. We notice that, although the original Higgs potential density $V_{0}$ given in (2.6) is purely sextic, the mass-deformed potential density $V_{m}$ obtained from adding the correction term $\Delta V_{0}$ given in (2.13) contains both quadratic and quartic terms, as that in the classical Chern-Simons-Higgs model [28,30].

It is evident that the associated Euler-Lagrange equations of the mass-deformed action

$$
\mathcal{A}_{\text {mass-deformed ABJM }}=\int\left\{\mathcal{L}_{\mathrm{CS}}+\mathcal{L}_{\text {kin }}-\left(V_{\text {ferm }}+\Delta V_{\text {ferm }}\right)-V_{m}\right\} \mathrm{d} x,
$$


are of course rather complicated. In the work of Kim, Kim, Kwon, and Nakajima [33, it is shown that these equations in their static limit and in the absence of fermions may be reduced into the following remarkable BPS system of equations

$$
\left.\begin{array}{rl}
\left(D_{1}-\mathrm{i} s D_{2}\right) Y^{1} & =0, \\
D_{i} Y^{A} & =0, \quad(A \neq 1, i=1,2), \\
D_{0} Y^{1}+\mathrm{i} s\left(\beta_{2}^{21}+\mu Y^{1}\right) & =0, \\
D_{0} Y^{2}-\mathrm{i} s\left(\beta_{1}^{12}+\mu Y^{2}\right) & =0, \\
D_{0} Y^{3}-\mathrm{i} s \beta_{1}^{13} & =0, \\
D_{0} Y^{4}-\mathrm{i} s \beta_{1}^{14} & =0, \\
\beta_{3}^{31} & =\beta_{4}^{41}=\beta_{3}^{21}+\mu Y^{1}, \\
\beta_{4}^{43} & =\mu Y^{3}, \\
\beta_{3}^{34} & =\mu Y^{4}, \\
\beta_{3}^{32}=\beta_{4}^{42}=\beta_{2}^{23}=\beta_{2}^{24} & =0, \\
\beta_{A}^{B C} & =0, \quad(A \neq B \neq C \neq A)
\end{array}\right\}
$$

where $s= \pm 1$ is a signature symbol to be specified later, coupled with the usual Gauss law constraints

$$
\begin{aligned}
\frac{\kappa}{2 \pi} B & \equiv \frac{\kappa}{2 \pi} F_{12}=j^{0}, \\
\frac{\kappa}{2 \pi} \hat{B} & \equiv \frac{\kappa}{2 \pi} \hat{F}_{12}=-\hat{j}^{0},
\end{aligned}
$$

where $j^{0}$ and $\hat{j}^{0}$ are two associated matrix-valued conserved currents given by the expressions

$$
\begin{aligned}
& j_{\mu}=\mathrm{i}\left(Y^{A} D_{\mu} Y_{A}^{\dagger}-D_{\mu} Y^{A} Y_{A}^{\dagger}\right), \\
& \hat{j}_{\mu}=\mathrm{i}\left(Y_{A}^{\dagger} D_{\mu} Y^{A}-D_{\mu} Y_{A}^{\dagger} Y^{A}\right) .
\end{aligned}
$$

In [33], it is demonstrated that the energy of the mass-deformed ABJM model has the topological lower bound

$$
E \geq \frac{1}{3} \mu\left|Q+2 R_{12}\right|
$$

where the topological charges $Q$ and $R_{12}$ are given by

$$
\begin{aligned}
Q & =\operatorname{Tr} \int j^{0} \mathrm{~d} x, \\
R_{12} & =\operatorname{Tr} \int J_{12}^{0} \mathrm{~d} x,
\end{aligned}
$$

for which the charge densities $j^{0}$ is defined in (2.18) and $J_{12}^{0}$ given by

$$
J_{12}^{0}=\mathrm{i}\left(Y^{1} D_{0} Y_{1}^{\dagger}-D_{0} Y^{1} Y_{1}^{\dagger}\right)-\mathrm{i}\left(Y^{2} D_{0} Y_{2}^{\dagger}-D_{0} Y^{2} Y_{2}^{\dagger}\right)
$$

and the integration is carried over the full two-dimensional spatial domain, and the lower bound (2.20) is attained by the solutions of the BPS system (2.15) coupled with the Gauss law constraints (2.16) -(2.17) so that $s$ is determined by the condition

$$
\left|Q+2 R_{12}\right|=s\left(Q+2 R_{12}\right)
$$


Thus it is important to understand the solutions of (2.15) -(2.17) which will be our goal in the present work.

To approach the system of equations (2.15)-2.17), Kim, Kim, Kwon, and Nakajima [33] take the following ansatz to represent the $N \times N$ matrices $Y^{A}=\left(Y_{i j}^{A}\right)$ :

$$
Y_{i j}^{1}=\delta_{i+1, j} \sqrt{\frac{k \mu}{2 \pi}} f_{i}, \quad Y_{i j}^{2}=\delta_{i j} \sqrt{\frac{k \mu}{2 \pi}} a_{i}, \quad Y^{3}=0, \quad Y^{4}=0,
$$

where $f_{i}(i=1, \ldots, N-1)$ are complex-valued functions and

$$
a_{i}=\sqrt{a^{2}+i-1}, \quad i=1, \ldots, N
$$

with $a \geq 0$ a constant. Within this ansatz, the $N \times N$ matrix-valued 'magnetic' fields become diagonal whose entries are given by 33 .

$$
B_{i j}=\hat{B}_{i j}=-2 s \delta_{i j} \mu^{2}\left(a^{2}+i-1\right)\left(\left|f_{i}\right|^{2}-\left|f_{i-1}\right|^{2}+1\right), \quad i, j=1, \ldots, N,
$$

where the convention $f_{0}=f_{N}=0$ is imposed. Using the complex-variable differentiation $\partial=$ $\frac{1}{2}\left(\partial_{1}-\mathrm{i} \partial_{2}\right), \bar{\partial}=\frac{1}{2}\left(\partial_{1}+\mathrm{i} \partial_{2}\right)$, and eliminating the gauge fields from the equations, it is shown in 33 ] that the BPS system (2.15)-(2.17) is reduced into the following system of $N-1(N \geq 3)$ coupled vortex equations:

$$
\begin{aligned}
\partial \bar{\partial} \ln \left|f_{1}\right|^{2}= & \mu^{2}\left(\left[2 a^{2}+1\right]\left|f_{1}\right|^{2}-\left[a^{2}+1\right]\left|f_{2}\right|^{2}-1\right) \\
\partial \bar{\partial} \ln \left|f_{i}\right|^{2}= & \mu^{2}\left(-\left[a^{2}+i-1\right]\left|f_{i-1}\right|^{2}+\left[2 a^{2}+2 i-1\right]\left|f_{i}\right|^{2}-\left[a^{2}+i\right]\left|f_{i+1}\right|^{2}-1\right), \\
& i=2, \ldots, N-2 \\
\partial \bar{\partial} \ln \left|f_{N-1}\right|^{2}= & \mu^{2}\left(-\left[a^{2}+N-2\right]\left|f_{N-2}\right|^{2}+\left[2 a^{2}+2 N-3\right]\left|f_{N-1}\right|^{2}-1\right)
\end{aligned}
$$

away from the zero points of $f_{1}, \ldots, f_{N-1}$, which are known to be the vortex points of the system.

Analyzing the structure of the system (2.15) as in [31, it may be seen that the zeros of the fields $f_{1}, \ldots, f_{N-1}$ are discrete and of integer multiplicities. Thus we can denote the sets of zeros of each $f_{i}$ by

$$
Z_{f_{i}}=\left\{p_{i, 1}, \ldots, p_{i, n_{i}}\right\}, \quad i=1, \ldots, N-1,
$$

such that the number of repetitions of any point $p$ among the set $Z_{f_{i}}=\left\{p_{i, s}\right\}(i=1, \ldots, N-1)$ takes account of the multiplicities of the zero.

We aim to prove that the prescribed sets of zeros given by (2.31) completely characterize the solution of (2.28)-(2.30). To be precise we note that the problem may be considered either over the full plane $\mathbb{R}^{2}$ under the natural boundary condition

$$
\lim _{|x| \rightarrow \infty}\left(\partial \bar{\partial} \ln \left|f_{i}\right|^{2}\right)(x)=0, \quad i=1, \ldots, N-1,
$$

or over a doubly periodic domain $\Omega$ so that the field configurations are subject to the 't Hooft periodic boundary condition [26, 48,53] for which periodicity is achieved modulo gauge transformations. We shall establish that in both cases solutions exist and are unique. 
Note that (2.28)-(2.30) only make sense when $N \geq 3$. When $N=2$ (the 'bottom' case with the gauge group $U(2) \times U(2))$, the system is a single equation [33]:

$$
\partial \bar{\partial} \ln |f|^{2}=\mu^{2}\left(\left[2 a^{2}+1\right]|f|^{2}-1\right),
$$

which has been well studied and existence and uniqueness results have been obtained [31,47,48].

We now proceed to state our main results.

Let $R$ be the $(N-1) \times(N-1)$ tridiagonal matrix given by

$$
\begin{aligned}
& R= \\
& \left(\begin{array}{cccccc}
2 a^{2}+1 & -\left(a^{2}+1\right) & 0 & \ldots & \ldots & 0 \\
-\left(a^{2}+1\right) & 2 a^{2}+3 & -\left(a^{2}+2\right) & 0 & \ldots & 0 \\
0 & -\left(a^{2}+2\right) & 2 a^{2}+5 & -\left(a^{2}+3\right) & \ldots & 0 \\
\vdots & & \ddots & \ddots & \ddots & \vdots \\
0 & & \ddots & -\left(a^{2}+N-3\right) & 2 a^{2}+2 N-5 & -\left(a^{2}+N-2\right) \\
0 & \ldots & & 0 & -\left(a^{2}+N-2\right) & 2 a^{2}+2 N-3
\end{array}\right) .
\end{aligned}
$$

We will see later that the matrix $R$ is positive definite. Denote the inverse of $R$ by $R^{-1}$. We shall also see that all entries of $R^{-1}$ are positive, i.e., $\left(R^{-1}\right)_{i j}>0, i, j=1, \ldots, N-1$. Write the eigenvalues of $R$ as $\lambda_{1}, \ldots, \lambda_{N-1}$, and set $\lambda_{0}$ to be the positive quantity

$$
\lambda_{0}=2 \min \left\{\lambda_{1}, \ldots, \lambda_{N-1}\right\} .
$$

Our main results are collectively summarized as follows.

Theorem 2.1 For any $a \geq 0, \mu>0$, consider the system of multiple vortex equations (2.28) -(2.30) for the field configuration $\left(f_{1}, \ldots, f_{N-1}\right)$ with the prescribed zero sets given by (2.31) such that each $f_{i}$ has $n_{i}$ arbitrarily distributed zeros $p_{i, 1}, \ldots, p_{i, n_{i}}, i=1, \ldots, N-1$.

(i) For the problem over the full plane $\mathbb{R}^{2}$, there exists a unique solution satisfying the boundary condition

$$
\left|f_{i}\right|^{2} \rightarrow r_{i}, \quad|x| \rightarrow \infty, \quad i=1, \ldots, N-1,
$$

which realizes the boundary condition (2.32), where

$$
r_{i} \equiv \sum_{j=1}^{N-1}\left(R^{-1}\right)_{i j}>0, \quad i=1, \ldots, N-1 .
$$

Moreover, this boundary condition is achieved exponentially fast at infinity,

$$
\left.|| f_{i}\right|^{2}-r_{i} \mid \leq C(\varepsilon) \mathrm{e}^{-2 \mu(1-\varepsilon) \sqrt{\lambda_{0}}|x|} \quad \text { as }|x| \rightarrow \infty, \quad i=1, \ldots, N-1,
$$

where $\varepsilon \in(0,1)$ is arbitrarily small, $C(\varepsilon)$ is a positive constant depending on $\varepsilon$, and $\lambda_{0}$ is defined by (2.35).

(ii) Over a doubly periodic domain $\Omega$, the problem admits a unique solution if and only if

$$
\pi \sum_{j=1}^{N-1}\left(R^{-1}\right)_{i j} n_{j}<\mu^{2}|\Omega| \sum_{j=1}^{N-1}\left(R^{-1}\right)_{i j}, \quad i=1, \ldots, N-1,
$$


hold simultaneously.

(iii) In both cases, there hold the quantized integrals

$$
\int\left(\sum_{j=1}^{N-1} R_{i j}\left|f_{j}\right|^{2}-1\right) \mathrm{d} x=-\frac{\pi n_{i}}{\mu^{2}}, \quad i=1, \ldots, N-1,
$$

where the integration is evaluated either over the full plane $\mathbb{R}^{2}$ or the doubly periodic domain $\Omega$.

It can be checked that (2.36) and (2.32) are equivalent. Thus, by virtue of (2.37), we see that the so-called non-topological solutions [12,14, 16, 29, 44] do not appear in the ABJM model [2, 33] considered here.

We now proceed to compute the associated flux.

As noticed in [33], the ansatz taken makes it consistent to assume that the gauge fields $A_{l}$ and $\hat{A}_{l}(l=1,2)$ are diagonal:

$$
A_{l}=\hat{A}_{l}=\operatorname{diag}\left\{a_{l}^{1}, \ldots, a_{l}^{N}\right\}, \quad l=1,2 .
$$

Thus, we may introduce the complex-valued variable

$$
A=\frac{1}{2}\left(A_{1}-\mathrm{i} A_{2}\right)=\operatorname{diag}\left\{\frac{1}{2}\left(a_{1}^{1}-\mathrm{i} a_{2}^{1}\right), \ldots, \frac{1}{2}\left(a_{1}^{N}-\mathrm{i} a_{2}^{N}\right)\right\} \equiv\left\{b_{1}, \ldots, b_{N}\right\},
$$

so that the matrix-valued 'magnetic' field $B=F_{12}$ becomes

$$
B=F_{12}=\partial_{1} A_{2}-\partial_{2} A_{1}=-2 \mathrm{i}(\partial \bar{A}-\bar{\partial} A)=-2 \mathrm{i} \operatorname{diag}\left\{\partial \bar{b}_{1}-\bar{\partial} b_{1}, \ldots, \partial \bar{b}_{N}-\bar{\partial} b_{N}\right\},
$$

which should not be confused with the group index used earlier. Hence, in view of (2.15), we have

$$
\mathrm{i} \bar{\partial} \ln f_{i-1}=\left(\bar{b}_{i-1}-\bar{b}_{i}\right), \quad i=2, \ldots, N,
$$

away from the zeros of $f_{i}(i=2, \ldots, N)$, where we have chosen $s=-1$ for definiteness. From (2.44), we obtain

$$
\frac{1}{4} \mathrm{i} \Delta \ln \left|f_{i-1}\right|^{2}=\mathrm{i} \partial \bar{\partial}\left(\ln f_{i-1}+\ln \bar{f}_{i-1}\right)=\left(\partial \bar{b}_{i-1}-\bar{\partial} b_{i-1}\right)-\left(\partial \bar{b}_{i}-\bar{\partial} b_{i}\right), \quad i=2, \ldots, N,
$$

again away from the zeros of the functions. Comparing (2.43) and (2.45), we see that $B \equiv$ $\operatorname{diag}\left\{B_{1}, \ldots, B_{N}\right\}$ over the same domain can be expressed as

$$
B_{i}=B_{i-1}-\frac{1}{2} \Delta \ln \left|f_{i-1}\right|^{2}=B_{1}-\frac{1}{2} \sum_{j=1}^{i-1} \Delta\left(\ln \left|f_{j}\right|^{2}\right), \quad i=2, \ldots, N
$$

where $B_{1}$ may be read off from (2.27). That is, $B_{1}=2 a^{2} \mu^{2}\left(\left|f_{1}\right|^{2}+1\right)$. Consequently, we get

$$
\operatorname{Tr}(B)=\sum_{i=1}^{N} B_{i}=N B_{1}-\frac{1}{2} \sum_{i=1}^{N-1}(N-i) \Delta \ln \left|f_{i}\right|^{2}
$$

In view of the equations (2.28) $-(2.30)$ and the quantized integral formulas stated in (2.40), we see that over the doubly periodic domain $\Omega$ the total 'magnetic' flux is

$$
-s \int_{\Omega} \operatorname{Tr}(B) \mathrm{d} x=2 N a^{2}\left(\left[1+\sum_{i=1}^{N-1}\left(R^{-1}\right)_{1 i}\right] \mu^{2}|\Omega|-\pi \sum_{i=1}^{N-1}\left(R^{-1}\right)_{1 i} n_{i}\right)+2 \pi \sum_{i=1}^{N-1}(N-i) n_{i},
$$


which is not quantized and depends on $|\Omega|$, unless $a=0$. Here we have switched on the dependence on the signature symbol $s= \pm 1$ in the flux formula for generality. So the flux over the full plane $\mathbb{R}^{2}$ diverges which leads to infinite energy as observed in 33 . Therefore, in order to avoid flux and energy divergence, it is of value to develop an existence theory for doubly periodic solutions when $a>0$ and of independent interest to spell out the existence theory separately when $a=0$. Indeed, when $a=0$, the matrix $R$ simplifies itself considerably so that the results can be stated in concrete terms explicitly as follows.

Theorem 2.2 For $a=0, \mu>0$, consider the system of vortex equations (2.28) -(2.30) for the field configuration $\left(f_{1}, \ldots, f_{N-1}\right)$ with the prescribed zero sets given by (2.31) such that each $f_{i}$ has $n_{i}$ arbitrarily distributed zeros $p_{i, 1}, \ldots, p_{i, n_{i}}, i=1, \ldots, N-1$.

(i) For the problem over the full plane $\mathbb{R}^{2}$, there exists a unique solution satisfying the boundary condition

$$
\left|f_{i}\right|^{2} \rightarrow(N-i), \quad|x| \rightarrow \infty, \quad i=1, \ldots, N-1 .
$$

Moreover, this boundary condition is achieved exponentially fast at infinity

$$
\left.|| f_{i}\right|^{2}-(N-i) \mid \leq C(\varepsilon) \mathrm{e}^{-2 \mu(1-\varepsilon) \sqrt{\lambda_{0}}|x|}, \quad i=1, \ldots, N-1,
$$

where $\varepsilon \in(0,1)$ is arbitrarily small, $C(\varepsilon)$ is a positive constant depending on $\varepsilon$, and $\lambda_{0}$ is defined by (2.35).

(ii) For the problem over a doubly periodic domain $\Omega$, there exists a unique solution if and only if the conditions

$$
\pi \sum_{j=i}^{N-1} \frac{1}{j} \sum_{l=1}^{j} n_{l}<\mu^{2}|\Omega|(N-i), \quad i=1, \ldots, N-1,
$$

hold simultaneously.

(iii) In both cases, there hold the quantized integrals

$$
\begin{aligned}
& \int\left(\left|f_{1}\right|^{2}-\left|f_{2}\right|^{2}-1\right) \mathrm{d} x=-\frac{\pi n_{1}}{\mu^{2}} \\
& \int\left(-[i-1]\left|f_{i-1}\right|^{2}+[2 i-1]\left|f_{i}\right|^{2}-i\left|f_{i+1}\right|^{2}-1\right) \mathrm{d} x=-\frac{\pi n_{i}}{\mu^{2}}, \quad i=2, \ldots, N-2, \\
& \int\left(-[N-2]\left|f_{N-2}\right|^{2}+[2 N-3]\left|f_{N-1}\right|^{2}-1\right) \mathrm{d} x=-\frac{\pi n_{N-1}}{\mu^{2}}
\end{aligned}
$$

where the integration is evaluated either over the full plane $\mathbb{R}^{2}$ or the doubly periodic domain $\Omega$.

To see the problem more transparently, we reformulate the system of equations (2.28)-(2.30) and Theorems 2.1 2.2 in terms of a new family of parameters and variables. For this purpose, we denote $N-1 \equiv m, \lambda \equiv 4 \mu^{2}$, and set

$$
u_{i}=\ln \left|f_{i}\right|^{2}, \quad i=1, \ldots, m
$$


Then, in view of the equations (2.28) $-(2.30)$ and the zero sets (2.31) we see that $u_{1}, \ldots, u_{m}$ satisfy the equations

$$
\begin{aligned}
\Delta u_{1}= & \lambda\left(\left[2 a^{2}+1\right] \mathrm{e}^{u_{1}}-\left[a^{2}+1\right] \mathrm{e}^{u_{2}}-1\right)+4 \pi \sum_{s=1}^{n_{1}} \delta_{p_{1, s}}, \\
\Delta u_{i}= & \lambda\left(-\left[a^{2}+i-1\right] \mathrm{e}^{u_{i-1}}+\left[2 a^{2}+2 i-1\right] \mathrm{e}^{u_{i}}-\left[a^{2}+i\right] \mathrm{e}^{u_{i+1}}-1\right)+4 \pi \sum_{s=1}^{n_{i}} \delta_{p_{i, s}}, \\
& i=2, \ldots m-1, \\
\Delta u_{m}= & \lambda\left(-\left[a^{2}+m-1\right] \mathrm{e}^{u_{m-1}}+\left[2 a^{2}+2 m-1\right] \mathrm{e}^{u_{m}}-1\right)+4 \pi \sum_{s=1}^{n_{m}} \delta_{p_{m, s}} .
\end{aligned}
$$

Let

$$
\left.\begin{array}{rl}
\mathbf{u} & =\left(u_{1}, \ldots, u_{m}\right)^{\tau}, \\
\mathbf{1} & =(1, \ldots, 1)^{\tau}, \\
\mathbf{U} & =\left(\mathrm{e}^{u_{1}}, \ldots, \mathrm{e}^{u_{m}}\right)^{\tau}, \\
\mathbf{s} & =\left(\sum_{s=1}^{n_{1}} \delta_{p_{1, s}}, \ldots, \sum_{s=1}^{n_{m}} \delta_{p_{m, s}}\right)^{\tau} \cdot
\end{array}\right\}
$$

Then the equations (2.56) $-(2.58)$ can be recast into its vector form

$$
\Delta \mathbf{u}=\lambda(R \mathbf{U}-\mathbf{1})+4 \pi \mathbf{s}
$$

Theorems 2.1 2.2 will be established through proving the following results for the equations (2.56) $-(2.58)$ or (2.60).

Theorem 2.3 For any $a \geq 0, \lambda>0$, consider the system of equations (2.56)-(2.58).

(i) There exists a unique solution over $\mathbb{R}^{2}$ satisfying the boundary conditions

$$
u_{i} \rightarrow \ln \left(\sum_{j=1}^{m}\left(R^{-1}\right)_{i j}\right) \equiv \ln r_{i}, \quad|x| \rightarrow \infty, \quad i=1, \ldots, m .
$$

Moreover, this solution satisfies the following exponential decay estimate at infinity:

$$
\sum_{i=1}^{m}\left(u_{i}(x)-\ln r_{i}\right)^{2} \leq C(\varepsilon) \mathrm{e}^{-(1-\varepsilon) \sqrt{\lambda \lambda_{0}}|x|}
$$

where $\varepsilon \in(0,1)$ is arbitrarily small, $C(\varepsilon)$ is a positive constant depending on $\varepsilon$, and $\lambda_{0}$ is defined by (2.35).

(ii) For the problem over a doubly periodic domain $\Omega$, a solution exists if and only if the following $m$ conditions

$$
4 \pi \sum_{j=1}^{m}\left(R^{-1}\right)_{i j} n_{j}<\lambda|\Omega| \sum_{j=1}^{m}\left(R^{-1}\right)_{i j}, \quad i=1, \ldots, m,
$$

hold simultaneously. Besides, if a solution exists, it must be unique. 
(iii) In both full plane and periodic domain cases, there hold the quantized integrals

$$
\int\left(\sum_{j=1}^{m} R_{i j} \mathrm{e}^{u_{j}}-1\right) \mathrm{d} x=-\frac{4 \pi n_{i}}{\lambda}, \quad i=1, \ldots, m,
$$

evaluated over the corresponding domain of consideration.

When $a=0$, we have the following explicit results.

Theorem 2.4 For $a=0, \lambda>0$, consider the system of equations (2.56)-(2.58).

(i) There exists a unique solution over $\mathbb{R}^{2}$ satisfying the boundary conditions

$$
u_{i} \rightarrow \ln (m-i+1), \quad|x| \rightarrow \infty, \quad i=1, \ldots, m .
$$

Moreover, this solution satisfies the following exponential decay estimate at infinity:

$$
\sum_{i=1}^{m}\left(u_{i}(x)-\ln (m-i+1)\right)^{2} \leq C(\varepsilon) \mathrm{e}^{-(1-\varepsilon) \sqrt{\lambda \lambda_{0}}|x|},
$$

where $\varepsilon \in(0,1)$ is small, $C(\varepsilon)>0$ depends on $\varepsilon$, and $\lambda_{0}$ is defined by (2.35).

(ii) There exists a solution over a doubly periodic domain $\Omega$ if and only if the conditions

$$
4 \pi \sum_{j=i}^{m} \frac{1}{j} \sum_{l=1}^{j} n_{l}<\lambda|\Omega|(m-i+1), \quad i=1, \ldots, m,
$$

are fulfilled simultaneously. Besides, if a solution exists, it must be unique.

(iii) In both cases, the quantized integrals

$$
\begin{aligned}
& \int\left(\mathrm{e}^{u_{1}}-\mathrm{e}^{u_{2}}-1\right) \mathrm{d} x=-\frac{4 \pi n_{1}}{\lambda}, \\
& \int\left(-[i-1] \mathrm{e}^{u_{i-1}}+[2 i-1] \mathrm{e}^{u_{i}}-i \mathrm{e}^{u_{i+1}}-1\right) \mathrm{d} x=-\frac{4 \pi n_{i}}{\lambda}, \quad i=1, \ldots, m-1, \\
& \int\left(-[m-1] \mathrm{e}^{u_{m-1}}+[2 m-1] \mathrm{e}^{u_{m}}-1\right) \mathrm{d} x=-\frac{4 \pi n_{m}}{\lambda},
\end{aligned}
$$

are valid over the corresponding domain of the problem.

In the subsequent sections, we prove Theorems 2.3 2.4 .

\section{Variational principle and solution to planar case}

In this section we establish the existence and uniqueness results for a solution of (2.56)-(2.58) over the full plane and derive the stated decay estimates for the solution. Unlike the problems studied in [35 37] which can be readily formulated variationally, the problem here needs more elaboration in order that its hidden variational structure be unveiled. For this purpose, we shall rely on the wellknown Cholesky decomposition theorem for positive-definite matrices. The variational structure to be recognized will allow us to prove the existence of a solution over the full plane as well as over a doubly periodic domain, although the present section is devoted to the planar case. Below we split our study into a few subsections. 


\subsection{Cholesky decomposition for the matrix $R$}

First, we observe that the symmetric matrix $R$ is positive definite. In fact, for any $a \geq 0$, it is easy to check that each leading principal minor of $R$ is positive. That is,

$$
R_{l} \equiv \operatorname{det}\left(\left[R_{i j}\right]_{l \times l}\right)>0, \quad i, j=1, \ldots, l, \quad l=1, \ldots, m .
$$

By the Cholesky decomposition theorem 23 , the matrix $R$ can be expressed as the product of a lower triangular matrix $L$ and its transpose, $R=L L^{\tau}, L=\left(L_{i j}\right)_{m \times m}$. Indeed, using the iteration scheme presented in [23], that is,

$$
\left.\begin{array}{rl}
L_{11} & =\sqrt{R_{11}}, \\
L_{i 1} & =\frac{R_{i 1}}{L_{11}}, i=2, \ldots, m, \\
L_{i i} & =\sqrt{R_{i i}-\sum_{j=1}^{i-1} L_{i j}^{2}}, \quad i=2, \ldots, m, \\
L_{i j} & =\frac{R_{i j}-\sum_{l=1}^{j-1} L_{i k} L_{j l}}{l_{j j}}, \quad i=j+1, \ldots, m, j=2, \ldots, m,
\end{array}\right\}
$$

we have

$$
\left.\begin{array}{rl}
L_{11} & =\sqrt{2 a^{2}+1}=\sqrt{R_{1}}, \quad L_{21}=-\frac{\left(a^{2}+1\right)}{\sqrt{R_{1}}}, \\
L_{i i-1} & =-\left(a^{2}+i-1\right) \sqrt{\frac{R_{i-2}}{R_{i-1}}}, \quad L_{i i}=\sqrt{\frac{R_{i}}{R_{i-1}}}, \quad i=2, \ldots, m, \quad L_{i j}=0, \quad 1 \leq j<i-1, \quad i=2, \ldots, m .
\end{array}\right\}
$$

Here and in the sequel, we follow the convention $R_{0}=1$. We have

$$
L=\left(\begin{array}{ccccc}
\sqrt{R_{1}} & 0 & 0 & \cdots & 0 \\
-\frac{a^{2}+1}{\sqrt{R_{1}}} & \sqrt{\frac{R_{2}}{R_{1}}} & 0 & \cdots & 0 \\
0 & -\left(a^{2}+2\right) \sqrt{\frac{R_{1}}{R_{2}}} & \sqrt{\frac{R_{3}}{R_{2}}} & \cdots & 0 \\
\vdots & \vdots & \vdots & \ddots & \vdots \\
\vdots & \vdots & \vdots & \vdots & \vdots \\
0 & 0 & \cdots & -\left(a^{2}+m-1\right) \sqrt{\frac{R_{m-2}}{R_{m-1}}} & \sqrt{\frac{R_{m}}{R_{m-1}}}
\end{array}\right)
$$

Furthermore, a simple calculation enables us to find the lower triangular matrix $L^{-1}$ with

$$
\left.\begin{array}{rl}
\left(L^{-1}\right)_{11} & =\frac{1}{\sqrt{R_{1}}}, \\
\left(L^{-1}\right)_{i j} & =\frac{\left(a^{2}+j\right) \cdots\left(a^{2}+i-1\right) R_{j-1}}{\sqrt{R_{i-1} R_{i}}}, \quad 1 \leq j \leq i-1, \quad i=2, \ldots, m, \\
\left(L^{-1}\right)_{i i} & =\sqrt{\frac{R_{i-1}}{R_{i}}}, \quad i=2, \ldots, m .
\end{array}\right\}
$$

By the expression of $L^{-1}$ we can compute the inverse of $R$ by the formula

$$
R^{-1}=\left(L L^{\tau}\right)^{-1}=\left(L^{-1}\right)^{\tau} L^{-1}
$$

from which we can see directly that, for any $a \geq 0$, all entries of $R^{-1}$ are positive: $\left(R^{-1}\right)_{i j}>$ $0, i, j=1, \ldots, m$, as claimed earlier. 


\subsection{Variational formulation}

Following [31], we introduce the background functions

$$
u_{0}^{i}=-\sum_{s=1}^{n_{i}} \ln \left(1+\nu\left|x-p_{i, s}\right|^{-2}\right), \quad i=1, \ldots, m,
$$

with $\nu>0$ being a parameter which should not be confused with the Lorentzian index used earlier. We see that $u_{0}^{i}$ satisfy

$$
\Delta u_{0}^{i}=4 \pi \sum_{s=1}^{n_{i}} \delta_{p_{i, s}}-g_{i}, \quad g_{i}=\sum_{s=1}^{n_{i}} \frac{4 \nu}{\left(\nu+\left|x-p_{i, s}\right|^{2}\right)^{2}} \quad i=1, \ldots, m .
$$

It is easy to see that

$$
g_{i} \in L\left(\mathbb{R}^{2}\right) \cap L^{2}\left(\mathbb{R}^{2}\right), \quad \int_{\mathbb{R}^{2}} g_{i} \mathrm{~d} x=4 \pi n_{i}, \quad i=1, \ldots, m .
$$

Since for any $a \geq 0$ each entry of $R^{-1}$ is positive, we have

$$
r_{i} \equiv \sum_{j=1}^{m}\left(R^{-1}\right)_{i j}>0, \quad i=1, \ldots, m
$$

Set $\mathbf{r}=\left(r_{1}, \ldots, r_{m}\right)^{\tau}$ and $u_{i}=u_{0}^{i}+\ln r_{i}+v_{i}, i=1, \ldots m, \mathbf{v}=\left(v_{1}, \ldots, v_{m}\right)^{\tau}, \mathbf{g}=\left(g_{1}, \ldots, g_{m}\right)^{\tau}$, $\mathbf{U}=\left(\mathrm{e}^{u_{0}^{1}+v_{1}}, \ldots, \mathrm{e}^{u_{0}^{m}+v_{m}}\right)^{\tau}$. Then the equations (2.56) $-(2.58)$ over $\mathbb{R}^{2}$ become

$$
\begin{aligned}
\Delta v_{1}= & \lambda\left(\left[2 a^{2}+1\right] r_{1} \mathrm{e}^{u_{0}^{1}+v_{1}}-\left[a^{2}+1\right] r_{2} \mathrm{e}^{u_{0}^{2}+v_{2}}-1\right)+g_{1} \\
\Delta v_{i}= & \lambda\left(-\left[a^{2}+i-1\right] r_{i-1} \mathrm{e}^{u_{0}^{i-1}+v_{i-1}}+\left[2 a^{2}+2 i-1\right] r_{i} \mathrm{e}^{u_{0}^{i}+v_{i}}\right. \\
& \left.-\left[a^{2}+i\right] r_{i+1} \mathrm{e}^{u_{0}^{i+1}+v_{i+1}}-1\right)+g_{i}, \quad i=2, \ldots m-1 \\
\Delta v_{m}= & \lambda\left(-\left[a^{2}+m-1\right] r_{m-1} \mathrm{e}^{u_{0}^{m-1}+v_{m-1}}+\left[2 a^{2}+2 m-1\right] r_{m} \mathrm{e}^{u_{0}^{m}+v_{m}}-1\right)+g_{m} .
\end{aligned}
$$

Or in its equivalent vector form, we have

$$
\Delta \mathbf{v}=\lambda R \operatorname{diag}\left\{r_{1}, \ldots, r_{m}\right\}(\mathbf{U}-\mathbf{1})+\mathbf{g},
$$

since $R \operatorname{diag}\left\{r_{1}, \ldots, r_{m}\right\} \mathbf{1}=\mathbf{1}$ by the definition of $r_{1}, \ldots, r_{m}$. Now we use the notation

$$
\mathbf{w}=\left(w_{1}, \ldots, w_{m}\right)^{\tau}, \quad \mathbf{h}=\frac{1}{\lambda} L^{-1} \mathbf{g}, \quad \mathbf{h}=\left(h_{1}, \ldots, h_{m}\right)^{\tau}
$$

and the transformation

$$
\mathbf{w}=L^{-1} \mathbf{v}=\left(\begin{array}{c}
\left(L^{-1}\right)_{11} v_{1} \\
\left(L^{-1}\right)_{21} v_{1}+\left(L^{-1}\right)_{22} v_{2} \\
\vdots \\
\left(L^{-1}\right)_{m 1} v_{1}+\cdots+\left(L^{-1}\right)_{m m} v_{m}
\end{array}\right),
$$


or

$$
\mathbf{v}=L \mathbf{w}=\left(\begin{array}{c}
L_{11} w_{1} \\
L_{21} w_{1}+L_{22} w_{2} \\
\vdots \\
L_{m m-1} w_{m-1}+L_{m m} w_{m}
\end{array}\right)
$$

Then the equations (3.11)-(3.13) take the form:

$$
\begin{aligned}
\Delta w_{1}= & \lambda\left(L_{11} r_{1}\left[\mathrm{e}^{u_{0}^{1}+L_{11} w_{1}}-1\right]+L_{21} r_{2}\left[\mathrm{e}^{u_{0}^{2}+L_{21} w_{1}+L_{22} w_{2}}-1\right]+h_{1}\right) \\
\Delta w_{i}= & \lambda\left(L_{i i} r_{i}\left[\mathrm{e}^{u_{0}^{i}+L_{i i-1} w_{i-1}+L_{i i} w_{i}}-1\right]\right. \\
& \left.+L_{i+1 i} r_{i+1}\left[\mathrm{e}^{u_{0}^{i+1}+L_{i+1 i} w_{i}+L_{i+1 i+1} w_{i+1}}-1\right]+h_{i}\right), \quad i=2, \ldots, m-1, \\
\Delta w_{m}= & \lambda\left(L_{m m} r_{m}\left[\mathrm{e}^{u_{0}^{m}+L_{m m-1} w_{m-1}+L_{m m} w_{m}}-1\right]+h_{m}\right) .
\end{aligned}
$$

Or in its vector form, we have

$$
\Delta \mathbf{w}=\lambda\left(L^{\tau} \operatorname{diag}\left\{r_{1}, \ldots, r_{m}\right\}[\mathbf{U}-\mathbf{1}]+\mathbf{h}\right) .
$$

It can now be checked to see that the equations (3.18)-(3.20) or (3.21) are the Euler-Lagrange equations of the functional

$$
\begin{aligned}
I(\mathbf{w})= & \frac{1}{2 \lambda} \sum_{i=1}^{m} \int_{\mathbb{R}^{2}}\left|\nabla w_{i}\right|^{2} \mathrm{~d} x+\sum_{i=1}^{m} \int_{\mathbb{R}^{2}} h_{i} w_{i} \mathrm{~d} x+\int_{\mathbb{R}^{2}} r_{1}\left(\mathrm{e}^{u_{0}^{1}+L_{11} w_{1}}-\mathrm{e}^{u_{0}^{1}}-L_{11} w_{1}\right) \mathrm{d} x \\
& +\sum_{i=2}^{m} \int_{\mathbb{R}^{2}} r_{i}\left(\mathrm{e}^{u_{0}^{i}+L_{i i-1} w_{i-1}+L_{i i} w_{i}}-\mathrm{e}^{u_{0}^{i}}-\left[L_{i i-1} w_{i-1}+L_{i i} w_{i}\right]\right) \mathrm{d} x .
\end{aligned}
$$

This is the variational principle we have aimed to unveil.

To facilitate the computation and analysis, it will be technically more convenient to rewrite the functional (3.22) as

$$
\begin{aligned}
I(\mathbf{w})= & \frac{1}{2 \lambda} \sum_{i=1}^{m} \int_{\mathbb{R}^{2}}\left|\nabla w_{i}\right|^{2} \mathrm{~d} x+\int_{\mathbb{R}^{2}} r_{1} \mathrm{e}^{u_{0}^{1}}\left(\mathrm{e}^{L_{11} w_{1}}-1-L_{11} w_{1}\right) \mathrm{d} x \\
& +\sum_{i=2}^{m} \int_{\mathbb{R}^{2}} r_{i} \mathrm{e}^{u_{0}^{i}}\left(\mathrm{e}^{L_{i i-1} w_{i-1}+L_{i i} w_{i}}-1-\left[L_{i i-1} w_{i-1}+L_{i i} w_{i}\right]\right) \mathrm{d} x \\
& +\sum_{i=1}^{m-1} \int_{\mathbb{R}^{2}}\left(L_{i i} r_{i}\left[\mathrm{e}^{u_{0}^{i}}-1\right]+L_{i+1 i} r_{i+1}\left[\mathrm{e}^{u_{0}^{i+1}}-1\right]+h_{i}\right) w_{i} \mathrm{~d} x \\
& +\int_{\mathbb{R}^{2}}\left(L_{m m} r_{m}\left[\mathrm{e}^{u_{0}^{m}}-1\right]+h_{m}\right) w_{m} \mathrm{~d} x
\end{aligned}
$$

which allows us to approach the problem in a similar manner as in [31,47] for the scalar situation, as we will do in the following. 
To proceed, we can compute to get

$$
\begin{aligned}
& (D I(\mathbf{w}))(\mathbf{w}) \\
& =\frac{1}{\lambda} \sum_{i=1}^{m} \int_{\mathbb{R}^{2}}\left|\nabla w_{i}\right|^{2} \mathrm{~d} x+\int_{\mathbb{R}^{2}}\left(L_{11} r_{1} \mathrm{e}^{u_{0}^{1}}\left[\mathrm{e}^{L_{11} w_{1}}-1\right]+L_{21} r_{2} \mathrm{e}^{u_{0}^{2}}\left[\mathrm{e}^{L_{21} w_{1}+L_{22} w_{2}}-1\right]\right) w_{1} \mathrm{~d} x \\
& +\sum_{i=2}^{m-1} \int_{\mathbb{R}^{2}}\left(L_{i i} r_{i} \mathrm{e}^{u_{0}^{i}}\left[\mathrm{e}^{L_{i i-1} w_{i-1}+L_{i i} w_{i}}-1\right]+L_{i+1 i} r_{i+1} \mathrm{e}^{u_{0}^{i+1}}\left[\mathrm{e}^{L_{i+1 i} w_{i}+L_{i+1 i+1} w_{i+1}}-1\right]\right) w_{i} \mathrm{~d} x \\
& +\int_{\mathbb{R}^{2}}\left(L_{m m} r_{m} \mathrm{e}^{u_{0}^{m}}\left[\mathrm{e}^{L_{m m-1} w_{m-1}+L_{m m} w_{m}}-1\right]\right) w_{m} \mathrm{~d} x \\
& +\sum_{i=1}^{m-1} \int_{\mathbb{R}^{2}}\left(L_{i i} r_{i}\left[\mathrm{e}^{u_{0}^{i}}-1\right]+L_{i+1 i} r_{i+1}\left[\mathrm{e}^{u_{0}^{i+1}}-1\right]+h_{i}\right) w_{i} \mathrm{~d} x \\
& +\int_{\mathbb{R}^{2}}\left(L_{m m} r_{m}\left[\mathrm{e}^{u_{0}^{m}}-1\right]+h_{m}\right) w_{m} \mathrm{~d} x \\
& =\frac{1}{\lambda} \sum_{i=1}^{m} \int_{\mathbb{R}^{2}}\left|\nabla w_{i}\right|^{2} \mathrm{~d} x+\int_{\mathbb{R}^{2}} r_{1}\left(\mathrm{e}^{u_{0}^{1}+L_{11} w_{1}}-1+\tilde{h}_{1}\right) L_{11} w_{1} \mathrm{~d} x \\
& +\sum_{i=2}^{m} \int_{\mathbb{R}^{2}} r_{i}\left(\mathrm{e}^{u_{0}^{i}+L_{i i-1} w_{i-1}+L_{i i} w_{i}}-1+\tilde{h}_{i}\right)\left(L_{i i-1} w_{i-1}+L_{i i} w_{i}\right) \mathrm{d} x
\end{aligned}
$$

where $\tilde{h}_{i}$ 's are some linear combinations of $h_{i}$ 's, also of $g_{i}$ 's. More precisely,

$$
\tilde{h}_{i}=\frac{1}{\lambda r_{i}} \sum_{j=1}^{m}\left(R^{-1}\right)_{j i} g_{j}, \quad i=1, \ldots, m
$$

Using the invertibility of the transformations (3.16) and (3.17), we see that there exist some positive constants $c_{1}$ and $c_{2}$ such that

$$
c_{1} \sum_{i=1}^{m} v_{i}^{2} \leq \sum_{i=1}^{m} w_{i}^{2} \leq c_{2} \sum_{i=1}^{m} v_{i}^{2}, \quad c_{1} \sum_{i=1}^{m}\left|\nabla v_{i}\right|^{2} \leq \sum_{i=1}^{m}\left|\nabla w_{i}\right|^{2} \leq c_{2} \sum_{i=1}^{m}\left|\nabla v_{i}\right|^{2} .
$$

Therefore, from (3.17), (3.24) and (3.26), we can obtain

$$
(D I(\mathbf{w}))(\mathbf{w}) \geq C_{0} \sum_{i=1}^{m} \int_{\mathbb{R}^{2}}\left|\nabla v_{i}\right|^{2} \mathrm{~d} x+\sum_{i=1}^{m} \int_{\mathbb{R}^{2}} r_{i}\left(\mathrm{e}^{u_{0}^{i}+v_{i}}-1+\tilde{h}_{i}\right) v_{i} \mathrm{~d} x,
$$

where $C_{0}$ is a positive constant.

To deal with the second term on the right hand side of (3.27), we follow the approach of [31]. We just need to estimate a typical term of the following form

$$
M(v)=\int_{\mathbb{R}^{2}}\left(\mathrm{e}^{u_{0}+v}-1+\tilde{h}\right) v \mathrm{~d} x .
$$

It is easy to see that $M(v)$ can be decomposed as $M(v)=M\left(v_{+}\right)+M\left(-v_{-}\right)$where $v_{+}=$ $\max \{v, 0\}, v_{-}=\max \{-v, 0\}$.

From the elementary inequality $\mathrm{e}^{t}-1 \geq t, t \in \mathbb{R}$ and the fact $u_{0}, \tilde{h} \in L^{2}\left(\mathbb{R}^{2}\right)$, we have

$$
M\left(v_{+}\right) \geq \int_{\mathbb{R}^{2}}\left(u_{0}+v_{+}+\tilde{h}\right) v_{+} \mathrm{d} x \geq \frac{1}{2} \int_{\mathbb{R}^{2}} v_{+}^{2} \mathrm{~d} x-C_{1},
$$


where and in the sequel we use $C$ to denote a generic positive constant.

To estimate $M\left(-v_{-}\right)$, we note the inequality $1-\mathrm{e}^{-t} \geq \frac{t}{1+t}, t \geq 0$, and obtain

$$
\begin{aligned}
M\left(-v_{-}\right) & =\int_{\mathbb{R}^{2}}\left(1-\mathrm{e}^{u_{0}-v_{-}}-\tilde{h}\right) v_{-} \mathrm{d} x \\
& =\int_{\mathbb{R}^{2}}\left(1-\tilde{h}-\mathrm{e}^{u_{0}}+\mathrm{e}^{u_{0}}\left[1-\mathrm{e}^{-v_{-}}\right]\right) v_{-} \mathrm{d} x \\
& \geq \int_{\mathbb{R}^{2}}\left(1-\tilde{h}-\mathrm{e}^{u_{0}}+\mathrm{e}^{u_{0}} \frac{v_{-}}{1+v_{-}}\right) v_{-} \mathrm{d} x \\
& =\int_{\mathbb{R}^{2}}\left(\left[1+v_{-}\right]\left[1-\tilde{h}-\mathrm{e}^{u_{0}}\right]+\mathrm{e}^{u_{0}} v_{-}\right) \frac{v_{-}}{1+v_{-}} \mathrm{d} x \\
& =\int_{\mathbb{R}^{2}}(1-\tilde{h}) \frac{v_{-}^{2}}{1+v_{-}} \mathrm{d} x+\int_{\mathbb{R}^{2}}\left(1-\mathrm{e}^{u_{0}}-\tilde{h}\right) \frac{v_{-}}{1+v_{-}} \mathrm{d} x \\
& \geq \frac{1}{2} \int_{\mathbb{R}^{2}} \frac{v_{-}^{2}}{1+v_{-}} \mathrm{d} x+\int_{\mathbb{R}^{2}}\left(1-\mathrm{e}^{u_{0}}-\tilde{h}\right) \frac{v_{-}}{1+v_{-}} \mathrm{d} x \\
& \geq \frac{1}{4} \int_{\mathbb{R}^{2}} \frac{v_{-}^{2}}{\left(1+v_{-}\right)^{2}} \mathrm{~d} x-C_{2},
\end{aligned}
$$

where we have used $\tilde{h} \leq \frac{1}{2}$, assured by taking $\nu$ sufficiently large, and the fact that both $\mathrm{e}^{u_{0}}-1$ and $\tilde{h}$ belong to $L^{2}\left(\mathbb{R}^{2}\right)$.

Then we see from (3.29) and (3.30) that

$$
M(v) \geq \frac{1}{4} \int_{\mathbb{R}^{2}} \frac{v^{2}}{(1+|v|)^{2}} \mathrm{~d} x-C .
$$

To proceed further, we need the following standard interpolation inequality over $W^{1,2}\left(\mathbb{R}^{2}\right)$ :

$$
\int_{\mathbb{R}^{2}} v^{4} \mathrm{~d} x \leq 2 \int_{\mathbb{R}^{2}} v^{2} \mathrm{~d} x \int_{\mathbb{R}^{2}}|\nabla v|^{2} \mathrm{~d} x, \quad \forall v \in W^{1,2}\left(\mathbb{R}^{2}\right) .
$$

Using (3.32), we have

$$
\begin{aligned}
& \left(\int_{\mathbb{R}^{2}}|v|^{2} \mathrm{~d} x\right)^{2} \\
& =\left(\int_{\mathbb{R}^{2}} \frac{|v|}{1+|v|}(1+|v|)|v| \mathrm{d} x\right)^{2} \\
& \leq \int_{\mathbb{R}^{2}} \frac{|v|^{2}}{(1+|v|)^{2}} \mathrm{~d} x \int_{\mathbb{R}^{2}}\left(|v|+|v|^{2}\right)^{2} \mathrm{~d} x \\
& \leq 4 \int_{\mathbb{R}^{2}} \frac{|v|^{2}}{(1+|v|)^{2}} \mathrm{~d} x \int_{\mathbb{R}^{2}}|v|^{2} \mathrm{~d} x\left(\int_{\mathbb{R}^{2}}|\nabla v|^{2} \mathrm{~d} x+1\right) \\
& \leq \frac{1}{2}\left(\int_{\mathbb{R}^{2}}|v|^{2} \mathrm{~d} x\right)^{2}+C\left(\left[\frac{|v|^{2}}{(1+|v|)^{2}} \mathrm{~d} x\right]^{4}+\left[\int_{\mathbb{R}^{2}}|\nabla v|^{2} \mathrm{~d} x\right]^{4}+1\right)
\end{aligned}
$$

which implies

$$
\|v\|_{2} \leq C\left(\int_{\mathbb{R}^{2}} \frac{|v|^{2}}{(1+|v|)^{2}} \mathrm{~d} x+\int_{\mathbb{R}^{2}}|\nabla v|^{2} \mathrm{~d} x+1\right)
$$


where and in the sequel we use $\|\cdot\|_{p}$ to denote the norm of the space $L^{p}\left(\mathbb{R}^{2}\right)$.

From (3.27) and (3.31), we obtain

$$
(D I(\mathbf{w}))(\mathbf{w}) \geq C_{2} \sum_{j=1}^{m} \int_{\mathbb{R}^{2}}\left(\left|\nabla v_{j}\right|^{2}+\frac{v_{j}^{2}}{\left(1+\left|v_{j}\right|\right)^{2}}\right) \mathrm{d} x-C_{3} .
$$

Then it follows from (3.26), (3.34) and (3.35) that

$$
(D I(\mathbf{w}))(\mathbf{w}) \geq C_{4} \sum_{j=1}^{m}\left\|w_{j}\right\|_{W^{1,2}\left(\mathbb{R}^{2}\right)}-C_{5} .
$$

By the coercive lower bound in (3.36), we can show that the functional $I$ defined by (3.22) admits a critical point. In fact, by (3.36), we can choose $\xi>0$ such that

$$
\inf \left\{D I(\mathbf{w})(\mathbf{w}) \mid\|\mathbf{w}\|_{W^{1,2}\left(\mathbb{R}^{2}\right)}=\xi\right\} \geq 1 .
$$

Since the functional $I$ is weakly lower semi-continuous on $W^{1,2}\left(\mathbb{R}^{2}\right)$, the minimization problem

$$
\eta_{0} \equiv \inf \left\{I(\mathbf{w}) \mid\|\mathbf{w}\|_{W^{1,2}\left(\mathbb{R}^{2}\right)} \leq \xi\right\}
$$

admits a solution, say, $\tilde{\mathbf{w}}$. Then, we can show that it must be an interior point. We argue by contradiction. Assume that $\|\tilde{\mathbf{w}}\|_{W^{1,2}\left(\mathbb{R}^{2}\right)}=\xi$. Then

$$
\lim _{t \rightarrow 0} \frac{I((1-t) \tilde{\mathbf{w}})-I(\tilde{\mathbf{w}})}{t}=\left.\frac{\mathrm{d}}{\mathrm{d} t} I((1-t) \tilde{\mathbf{w}})\right|_{t=0}=-(D I(\tilde{\mathbf{w}}))(\tilde{\mathbf{w}}) \leq-1
$$

Therefore, if $t>0$ is sufficiently small, letting $\tilde{\mathbf{w}}^{t}=(1-t) \tilde{\mathbf{w}}$, we can obtain

$$
I\left(\tilde{\mathbf{w}}^{t}\right)<I(\tilde{\mathbf{w}})=\eta_{0}, \quad\left\|\tilde{\mathbf{w}}^{t}\right\|_{W^{1,2}\left(\mathbb{R}^{2}\right)}=(1-t) \xi<\xi,
$$

which contradicts the definition of $\eta_{0}$. Hence, $\tilde{\mathbf{w}}$ must be an interior critical point for the problem (3.38). As a result, it is a critical point of the functional $I$. Since the functional $I$ is strictly convex, this critical point must be unique.

\subsection{Asymptotic behavior of the solution at infinity}

Here we study the asymptotic behavior of the solution obtained above. Noting that $\mathbf{w} \in W^{1,2}\left(\mathbb{R}^{2}\right)$, by the well-known inequality

$$
\left\|\mathrm{e}^{v}-1\right\|_{2}^{2} \leq C_{1} \exp \left(C_{2}\|v\|_{W^{1,2}\left(\mathbb{R}^{2}\right)}^{2}\right), \quad \forall v \in W^{1,2}\left(\mathbb{R}^{2}\right)
$$

where $C_{1}, C_{2}$ are some positive constants, we see that the right-hand sides of the equations (3.18)(3.20) all belong to $L^{2}\left(\mathbb{R}^{2}\right)$. Using the standard elliptic $L^{2}$-estimates, we have $w_{j} \in W^{2,2}\left(\mathbb{R}^{2}\right)$, which implies $w_{j} \rightarrow 0$ as $|x| \rightarrow \infty, j=1, \ldots, m$. By the transformation (3.17), we see that $v_{j} \rightarrow 0$ as $|x| \rightarrow \infty$, which implies the desired boundary condition $u_{j} \rightarrow \ln r_{j}$ as $|x| \rightarrow \infty, j=1, \ldots, m$.

Next we show that $\left|\nabla w_{j}\right| \rightarrow 0$ as $|x| \rightarrow \infty, j=1, \ldots, m$. A typical term of the right hand sides of (3.18)-(3.20) can be rewritten as

$$
\mathrm{e}^{u_{0}^{j}+L_{j j-1} w_{j-1}+L_{j j} w_{j}}-1=\left(\mathrm{e}^{u_{0}^{j}}-1\right) \mathrm{e}^{L_{j j-1} w_{j-1}+L_{j j} w_{j}}+\left(\mathrm{e}^{L_{j j-1} w_{j-1}+L_{j j} w_{j}}-1\right),
$$


which belongs to $L^{p}\left(\mathbb{R}^{2}\right)$ for any $p>2$ due to the embedding $W^{1,2}\left(\mathbb{R}^{2}\right) \subset L^{p}\left(\mathbb{R}^{2}\right)$ and the definition of $u_{0}^{j}$. Therefore all the right-hand-side terms of (3.18)-(3.20) lie in $L^{p}\left(\mathbb{R}^{2}\right)$, for any $p>2$. Then the elliptic $L^{p}$-estimates imply $w_{j} \in W^{2, p}\left(\mathbb{R}^{2}\right)$ for any $p>2, j=1, \ldots, m$. Consequently, we have $\left|\nabla w_{j}\right| \rightarrow 0$ as $|x| \rightarrow \infty, j=1, \ldots m$. That is, $\left|\nabla u_{j}\right| \rightarrow 0$ as $|x| \rightarrow \infty, j=1, \ldots m$.

Now we establish the exponential decay rate of the solutions at infinity. To this end, we consider the equations (2.56)-(2.58) or (2.60) over an exterior domain

$$
D_{\rho}=\left\{x \in \mathbb{R}^{2}|\quad| x \mid>\rho\right\},
$$

where $\rho>0$ satisfies

$$
\rho>\max \left\{\left|p_{i, s}\right| \mid i=1, \ldots, m, s=1, \ldots, n_{i}\right\} .
$$

For convenience, we consider the system of equations (2.60) over $D_{\rho}$. Recall that $\mathbf{r}=R^{-1} \mathbf{1}$. Hence we may rewrite (2.60) in $D_{\rho}$ as

$$
\Delta \mathbf{u}=\lambda R(\mathbf{U}-\mathbf{r})=\lambda R \mathbf{v}+\lambda R(\mathbf{U}-\mathbf{r}-\mathbf{v})
$$

where vector $\mathbf{v}$ is to be determined.

Since the matrix $R$ is positive definite, there is an orthogonal matrix $O$ such that

$$
O^{\tau} R O=\operatorname{diag}\left\{\lambda_{1}, \ldots, \lambda_{m}\right\}, \quad \min \left\{\lambda_{1}, \ldots, \lambda_{m}\right\}>0 .
$$

Now apply $O^{\tau}$ in (3.45) and set

$$
\tilde{\mathbf{u}}=O^{\tau} \mathbf{v}, \quad \mathbf{v}=\left(u_{1}-\ln r_{1}, \ldots, u_{m}-\ln r_{m}\right)^{\tau} .
$$

Then we have

$$
\Delta \tilde{\mathbf{u}}=\lambda \operatorname{diag}\left\{\lambda_{1}, \ldots, \lambda_{m}\right\} \tilde{\mathbf{u}}+\lambda O^{\tau} R(\mathbf{U}-\mathbf{r}-\mathbf{v}) .
$$

Note that, since $\mathbf{U} \rightarrow \mathbf{r}$ as $|x| \rightarrow \infty$, we have $\mathbf{U}-\mathbf{r}=E(x) \mathbf{v}$ where $E(x)$ is an $m \times m$ diagonal matrix so that $E(x) \rightarrow I_{m}$ (the $m \times m$ identity matrix) as $|x| \rightarrow \infty$. This observation leads us to rewrite (3.48) as

$$
\Delta \tilde{\mathbf{u}}=\lambda \operatorname{diag}\left\{\lambda_{1}, \ldots, \lambda_{m}\right\} \tilde{\mathbf{u}}+\lambda P(x) \tilde{\mathbf{u}},
$$

where $P(x)$ is an $m \times m$ matrix which vanishes at infinity. As a consequence of (3.49), we obtain

$$
\Delta|\tilde{\mathbf{u}}|^{2} \geq 2 \tilde{\mathbf{u}}^{\tau} \Delta \tilde{\mathbf{u}} \geq \lambda \lambda_{0}|\tilde{\mathbf{u}}|^{2}-b(x)|\tilde{\mathbf{u}}|^{2}
$$

with $b(x) \rightarrow 0$ as $|x| \rightarrow \infty$.

Then, for any $\varepsilon \in(0,1)$, we can find a suitably large $\rho_{\varepsilon} \geq \rho$ such that

$$
\Delta|\tilde{\mathbf{u}}|^{2} \geq\left(1-\frac{\varepsilon}{2}\right) \lambda \lambda_{0}|\tilde{\mathbf{u}}|^{2}, \quad x \in D_{\rho_{\varepsilon}} .
$$

We will use a comparison function, say $\eta$, of the form

$$
\eta=C \mathrm{e}^{-\sigma|x|}, \quad|x|>0, \quad C, \sigma \in \mathbb{R}, \quad C, \sigma>0 .
$$

Then $\Delta \eta=\sigma^{2} \eta-\frac{\sigma}{|x|} \eta$. Thus, in view of (3.51), we have

$$
\Delta\left(|\tilde{\mathbf{u}}|^{2}-\eta\right) \geq\left(1-\frac{\varepsilon}{2}\right) \lambda \lambda_{0}|\tilde{\mathbf{u}}|^{2}-\sigma^{2} \eta, \quad|x| \geq \rho_{\varepsilon} .
$$


We take the obvious choice $\sigma^{2}=\left(1-\frac{\varepsilon}{2}\right) \lambda \lambda_{0}$ which gives us $\Delta\left(|\tilde{\mathbf{u}}|^{2}-\eta\right) \geq \sigma^{2}\left(|\tilde{\mathbf{u}}|^{2}-\eta\right),|x| \geq \rho_{\varepsilon}$. Choose $C$ in (3.52) large so that $|\tilde{\mathbf{u}}|^{2}-\eta \leq 0$ for $|x|=\rho_{\varepsilon}$. Hence, using the fact that $|\tilde{\mathbf{u}}| \rightarrow 0$ as $|x| \rightarrow \infty$ and the maximum principle, we see that $|\tilde{\mathbf{u}}|^{2} \leq \eta$ for $|x| \geq \rho_{\varepsilon}$. So the estimate

$$
|\tilde{\mathbf{u}}|^{2} \leq C(\varepsilon) \mathrm{e}^{-(1-\varepsilon) \sqrt{\lambda \lambda_{0}}|x|}, \quad|x| \geq \rho_{\varepsilon},
$$

follows since $(1-\varepsilon)^{2}<\left(1-\frac{\varepsilon}{2}\right)$ for any $\varepsilon \in(0,1)$. Therefore the desired exponential decay rate (2.62) is established.

To get the quantized integrals, we need to establish the exponential decay rate for the derivatives.

Let $\partial$ denote any of the two derivatives $\partial_{1}$ and $\partial_{2}$. Define

$$
\mathbf{v}=\left(\partial u_{1}, \ldots, \partial u_{m}\right)^{\tau}, \quad P=\operatorname{diag}\left\{r_{1}, \ldots, r_{m}\right\}, \quad Q=\operatorname{diag}\left\{\mathrm{e}^{u_{1}}-r_{1}, \ldots, \mathrm{e}^{u_{m}}-r_{m}\right\} .
$$

Then differentiating (2.60) in $D_{\rho}$, we have

$$
\Delta \mathbf{v}=\lambda R P \mathbf{v}+\lambda R Q \mathbf{v}
$$

Let $O$ be as before and set

$$
P \mathbf{v}=O \tilde{\mathbf{u}}, \quad f=\mathbf{v}^{\tau} P \mathbf{v} .
$$

Then by (3.56) and the fact that $r_{1}, \ldots, r_{m}>0$, we obtain

$$
\begin{aligned}
\Delta f & \geq 2 \mathbf{v}^{\tau} P \Delta \mathbf{v}=2 \lambda \mathbf{v}^{\tau} P R P \mathbf{v}+2 \lambda \mathbf{v}^{\tau} P R Q \mathbf{v} \\
& \geq \lambda \lambda_{0}|\tilde{\mathbf{u}}|^{2}+2 \lambda \mathbf{v}^{\tau} P R Q \mathbf{v}=\lambda \lambda_{0} \mathbf{v}^{\tau} P^{2} \mathbf{v}+2 \lambda \mathbf{v}^{\tau} P R Q \mathbf{v} \\
& \geq \lambda \lambda_{0} r_{0} f-b(x) f
\end{aligned}
$$

where $r_{0}=\min \left\{r_{1}, \ldots, r_{m}\right\}$ and $b(x) \rightarrow 0$ as $|x| \rightarrow \infty$. Then, as discussed previously, we can conclude that for any $\varepsilon \in(0,1)$, there is a positive constant $C(\varepsilon)>0$, such that

$$
f \leq C(\varepsilon) \mathrm{e}^{-(1-\varepsilon) \sqrt{\lambda \lambda_{0} r_{0}}|x|}
$$

when $|x|$ is sufficiently large. Thus we get the following exponential decay rate near infinity:

$$
\sum_{i=1}^{m}\left|\nabla u_{i}(x)\right|^{2} \leq C(\varepsilon) \mathrm{e}^{-(1-\varepsilon) \sqrt{\lambda \lambda_{0} r_{0}}|x|} .
$$

We can now calculate the quantized integrals (2.64) stated in Theorem 2.3 for the planar case.

Using (3.7), (3.8), and the exponential decay property of $\left|\nabla u_{i}\right|$ 's in (3.60), we conclude that $\left|\nabla v_{i}\right|$ 's vanish at infinity at least at the rate $|x|^{-3}$. Thus, using the divergence theorem, we have

$$
\int_{\mathbb{R}^{2}} \Delta v_{i} \mathrm{~d} x=0, \quad i=1, \ldots, m
$$

Consequently, integrating the equations (3.11)-(3.13) over $\mathbb{R}^{2}$, and applying (3.9) and (3.61), we obtain the desired results stated in (2.64).

We next turn our attention to the compact case. 


\section{Doubly periodic case}

In this section we consider the equations (2.56)-(2.58) over a doubly periodic domain $\Omega$.

Let $u_{0}^{i}$ be a solution of the problem (see [3])

$$
\Delta u_{0}^{i}=-\frac{4 \pi n_{i}}{|\Omega|}+4 \pi \sum_{s=1}^{n_{i}} \delta_{p_{i, s}}, \quad x \in \Omega, \quad i=1, \ldots, m .
$$

Set $u_{i}=u_{0}^{i}+v_{i}, i=1, \ldots, m$. Then equations (2.56) $-(2.58)$ become

$$
\begin{aligned}
\Delta v_{1}= & \lambda\left(\left[2 a^{2}+1\right] \mathrm{e}^{u_{0}^{1}+v_{1}}-\left[a^{2}+1\right] \mathrm{e}^{u_{0}^{2}+v_{2}}-1\right)+\frac{4 \pi n_{1}}{|\Omega|}, \\
\Delta v_{i}= & \lambda\left(-\left[a^{2}+i-1\right] \mathrm{e}^{u_{0}^{i-1}+v_{i-1}}+\left[2 a^{2}+2 i-1\right] \mathrm{e}^{u_{0}^{i}+v_{i}}-\left[a^{2}+i\right] \mathrm{e}^{u_{0}^{i+1}+v_{i+1}}-1\right) \\
& +\frac{4 \pi n_{i}}{|\Omega|}, \quad i=2, \ldots, m-1, \\
\Delta v_{m}= & \lambda\left(-\left[a^{2}+m-1\right] \mathrm{e}^{u_{0}^{m-1}+v_{m-1}}+\left[2 a^{2}+2 m-1\right] \mathrm{e}^{u_{0}^{m}+v_{m}}-1\right)+\frac{4 \pi n_{m}}{|\Omega|},
\end{aligned}
$$

or equivalently in its vector form

$$
\Delta \mathbf{v}=\lambda(R \mathbf{U}-\mathbf{1})+\frac{4 \pi}{|\Omega|} \mathbf{n},
$$

with $\mathbf{U}=\left(\mathrm{e}^{u_{0}^{1}+v_{1}}, \ldots, \mathrm{e}^{u_{0}^{m}+v_{m}}\right)^{\tau}, \mathbf{v}=\left(v_{1}, \ldots v_{m}\right)^{\tau}, \mathbf{1}=(1, \ldots, 1)^{\tau}, \mathbf{n}=\left(n_{1}, \ldots, n_{m}\right)^{\tau}$.

Integrating the equations (4.2)-(4.4) or (4.5) over $\Omega$, we can obtain the natural constraints

$$
R \int_{\Omega} \mathbf{U} \mathrm{d} x=|\Omega| \mathbf{1}-\frac{4 \pi}{\lambda} \mathbf{n} .
$$

We may rewrite (4.6) more conveniently as

$$
\int_{\Omega} \mathbf{U d} x=|\Omega| R^{-1} \mathbf{1}-\frac{4 \pi}{\lambda} R^{-1} \mathbf{n} \equiv \mathbf{K},
$$

or in its component form

$$
\int_{\Omega} \mathrm{e}^{u_{0}^{i}+v_{i}} \mathrm{~d} x=|\Omega| \sum_{j=1}^{m}\left(R^{-1}\right)_{i j}-\frac{4 \pi}{\lambda} \sum_{j=1}^{m}\left(R^{-1}\right)_{i j} n_{j}=K_{i}, \quad i=1, \ldots, m,
$$

where and in what follows we use the notation $\mathbf{K}=\left(K_{1}, \ldots, K_{m}\right)^{\tau}$. Therefore, we see that if a solution exists, then $K_{i}>0, i=1, \ldots, m$. As a result, we get the necessity of the condition (2.61).

In what follows we show that the condition (2.61) is also sufficient for the existence of a solution to (4.2) - (4.4) by variational methods.

We will work on the Sobolev spaces $W^{1,2}(\Omega)$, which is composed of scalar- or vector-valued $\Omega$-periodic $L^{2}$-functions whose derivatives also belong to $L^{2}(\Omega)$. For the scalar case we have the decomposition

$$
W^{1,2}(\Omega)=\mathbb{R} \oplus \dot{W}^{1,2}(\Omega)
$$

where

$$
\dot{W}^{1,2}(\Omega)=\left\{w \in W^{1,2}(\Omega) \mid \int_{\Omega} w \mathrm{~d} x=0\right\},
$$


is a closed subspace of $W^{1,2}(\Omega)$. Then for any $u \in W^{1,2}(\Omega)$, we have

$$
u=\underline{u}+\dot{u}, \quad \underline{u} \in \mathbb{R}, \quad \dot{u} \in \dot{W}^{1,2}(\Omega)
$$

We will use the well-known Trudinger-Moser inequality [3, 19]

$$
\int_{\Omega} \mathrm{e}^{u} \mathrm{~d} x \leq C \exp \left(\frac{1}{16 \pi} \int_{\Omega}|\nabla u|^{2} \mathrm{~d} x\right), \quad \forall u \in \dot{W}^{1,2}(\Omega),
$$

which is important for our estimate, although the analysis does not depend on the optimality of the embedding constant.

As in the planar case, to formulate the problem in a variational structure, we use the transformation (3.16) or (3.17). Then the equations (4.2)-(4.4) become

$$
\begin{aligned}
\Delta w_{1}= & \lambda\left(L_{11} \mathrm{e}^{u_{0}^{1}+L_{11} w_{1}}+L_{21} \mathrm{e}^{u_{0}^{2}+L_{21} w_{1}+L_{22} w_{2}}-b_{1}\right), \\
\Delta w_{i}= & \lambda\left(L_{i i} \mathrm{e}^{u_{0}^{i}+L_{i i-1} w_{i-1}+L_{i i} w_{i}}+L_{i+1 i} \mathrm{e}^{u_{0}^{i+1}+L_{i+1 i} w_{i}+L_{i+1 i+1} w_{i+1}}-b_{i}\right), \\
& i=2, \ldots, m-1 \\
\Delta w_{m}= & \lambda\left(L_{m m} \mathrm{e}^{u_{0}^{m}+L_{m m-1} w_{m-1}+L_{m m} w_{m}}-b_{m}\right),
\end{aligned}
$$

whose vector form is

$$
\Delta \mathbf{w}=\lambda\left(L^{\tau} \mathbf{U}-\mathbf{b}\right)
$$

where

$$
\mathbf{b}=\left(b_{1}, \ldots, b_{m}\right)^{\tau} \equiv L^{-1} \mathbf{1}-\frac{4 \pi}{\lambda|\Omega|} L^{-1} \mathbf{n}
$$

As before, we can check to see that the equations (4.13)-(4.15) are the Euler-Lagrange equations of the functional

$$
I(\mathbf{w})=\frac{1}{2 \lambda} \sum_{i=1}^{m} \int_{\Omega}\left|\nabla w_{i}\right|^{2} \mathrm{~d} x+\int_{\Omega}\left(\mathrm{e}^{u_{0}^{1}+L_{11} w_{1}}+\sum_{i=2}^{m} \mathrm{e}^{u_{0}^{i}+L_{i i-1} w_{i-1}+L_{i i} w_{i}}\right) \mathrm{d} x-\sum_{i=1}^{m} \int_{\Omega} b_{i} w_{i} \mathrm{~d} x
$$

We shall now engage ourselves in a direct minimization procedure, initiated in [35, to find a critical point of the functional (4.18).

When $\mathbf{w} \in W^{1,2}(\Omega)$, by the Trudinger-Moser inequality (4.12), we see that the functional defined by (4.18) is a $C^{1}$-functional and lower semi-continuous with respect to the weak topology of $W^{1,2}(\Omega)$.

Using the decomposition formula (4.11), we obtain

$$
\begin{aligned}
I(\mathbf{w})-\frac{1}{2 \lambda} \sum_{i=1}^{m}\left\|\nabla \dot{w}_{i}\right\|_{2}^{2} & =\int_{\Omega}\left(\mathrm{e}^{u_{0}^{1}+L_{11} w_{1}}+\sum_{i=2}^{m} \mathrm{e}^{u_{0}^{i}+L_{i i-1} w_{i-1}+L_{i i} w_{i}}\right) \mathrm{d} x-|\Omega| \mathbf{b}^{\tau} \underline{\mathbf{w}} \\
& =\sum_{i=1}^{m} \int_{\Omega} \mathrm{e}^{u_{0}^{i}+v_{i}} \mathrm{~d} x-\mathbf{K}^{\tau} \underline{\mathbf{v}} \\
& =\sum_{i=1}^{m} \int_{\Omega} \mathrm{e}^{u_{0}^{i}+\dot{v}_{i}+\underline{v}_{i}} \mathrm{~d} x-\sum_{i=1}^{m} K_{i} \underline{v}_{i},
\end{aligned}
$$


where we have used $|\Omega| \mathbf{b}^{\tau} \underline{\mathbf{w}}=\mathbf{K}^{\tau} \underline{\mathbf{v}}$ in view of (4.7) and (4.17).

By Jensen's inequality, we see that

$$
\begin{aligned}
\int_{\Omega} \mathrm{e}^{u_{0}^{i}+\dot{v}_{i}+\underline{v}_{i}} \mathrm{~d} x & \geq|\Omega| \exp \left(\frac{1}{|\Omega|} \int_{\Omega}\left(u_{0}^{i}+\dot{v}_{i}+\underline{v}_{i}\right) \mathrm{d} x\right) \\
& =|\Omega| \exp \left(\frac{1}{|\Omega|} \int_{\Omega} u_{0}^{i} \mathrm{~d} x\right) \mathrm{e}^{\underline{v}_{i}} \equiv \sigma_{i} \mathrm{e}^{-}, \quad i=1, \ldots, m .
\end{aligned}
$$

Using the condition (2.63), we have $K_{i}>0, i=1, \ldots, m$. Then, combining (4.19) and (4.20), we have

$$
\begin{aligned}
I(\mathbf{w})-\frac{1}{2 \lambda} \sum_{i=1}^{m} \int_{\Omega}\left|\nabla \dot{w}_{i}\right|^{2} \mathrm{~d} x & \geq \sum_{i=1}^{m}\left(\sigma_{i} \mathrm{e}^{\underline{v}}-K_{i} \underline{v}_{i}\right) \\
& \geq \sum_{i=1}^{m} K_{i} \ln \frac{\sigma_{i}}{K_{i}}
\end{aligned}
$$

Hence, from (4.21), we see that the functional $I$ is bounded from below and the minimization problem

$$
\eta_{0} \equiv \inf \left\{I(\mathbf{w}) \mid \mathbf{w} \in W^{1,2}(\Omega)\right\}
$$

is well-defined.

Let $\left\{\mathbf{w}^{(\ell)}\right\}=\left\{\left(w_{1}^{(\ell)}, \ldots, w_{m}^{(\ell)}\right)\right\}$ be a minimizing sequence of (4.22). It is easy to see that the function $f(t)=\sigma e^{t}-\eta t$, where $\sigma, \eta$ are positive constants, satisfies the property that $f(t) \rightarrow$ $\infty$ as $t \rightarrow \pm \infty$. Thus, we conclude from (4.21) that $\left\{\underline{v}_{i}^{(\ell)}\right\}(i=1, \ldots, m)$ are bounded. As a result, $\left\{\underline{w}_{i}^{(\ell)}\right\}(i=1, \ldots, m)$ are bounded. Therefore, the sequences $\left\{\underline{w}_{i}^{(\ell)}\right\}(i=1, \ldots, m)$ admit convergent subsequences, which are still denoted by $\left\{\underline{w}_{i}^{(\ell)}\right\}(i=1, \ldots, m)$ for convenience. Then, there exist $m$ real numbers $\underline{w}_{1}^{(\infty)}, \ldots, \underline{w}_{m}^{(\infty)} \in \mathbb{R}$ such that $\underline{w}_{i}^{(\ell)} \rightarrow \underline{w}_{i}^{(\infty)}(i=1, \ldots, m)$, as $\ell \rightarrow \infty$.

Using (4.21) again, we infer that $\left\{\nabla \dot{w}_{i}^{(\ell)}\right\}(i=1, \ldots, m)$ are bounded in $L^{2}(\Omega)$. Therefore, it follows from the Poincaré inequality that the sequences $\left\{\dot{w}_{i}^{(\ell)}\right\}(i=1, \ldots, m)$ are bounded in $W^{1,2}(\Omega)$. Consequently, the sequences $\left\{\dot{w}_{i}^{(\ell)}\right\}(j=1, \ldots, m)$ admit weakly convergent subsequences, which are still denoted by $\left\{\dot{w}_{i}^{(\ell)}\right\}(i=1, \ldots, m)$ for convenience. Then, there exist $m$ functions $\dot{w}_{i}^{(\infty)} \in W^{1,2}(\Omega)(i=1, \ldots, m)$ such that $\dot{w}_{i}^{(\ell)} \rightarrow \dot{w}_{i}^{(\infty)}$ weakly in $W^{1,2}(\Omega)$ as $\ell \rightarrow \infty(i=1, \ldots, m)$. Of course, $\dot{w}_{i}^{(\infty)} \in \dot{W}^{1,2}(\Omega)(i=1, \ldots, m)$.

Set $w_{i}^{(\infty)}=\underline{w}_{i}^{(\infty)}+\dot{w}_{i}^{(\infty)}(i=1, \ldots, m)$, which are all in $W^{1,2}(\Omega)$ naturally. Then, the above convergence result implies $w_{i}^{(\ell)} \rightarrow w_{i}^{(\infty)}(i=1, \ldots, m)$ weakly in $W^{1,2}(\Omega)$ as $\ell \rightarrow \infty$. Noting that the functional $I(\mathbf{w})$ is weakly lower semi-continuous in $W^{1,2}(\Omega)$, we conclude that $\left(w_{1}^{(\infty)}, \ldots, w_{m}^{(\infty)}\right)$ is a solution of the minimization problem (4.22) and is a critical point of $I(\mathbf{w})$. As a critical point of $I(\mathbf{w})$, it satisfies the equations (4.13)-(4.15).

Since the matrix $R$ is positive definite, it is easy to check that $I(\mathbf{w})$ is strictly convex over $W^{1,2}(\Omega)$. As a result, the functional $I(\mathbf{w})$ has at most one critical point in $W^{1,2}(\Omega)$, which implies the uniqueness of the solution to the equations (4.13)-(4.15). 
As in [35, we briefly remark that we can also find a critical point of the functional $I$ by a constrained minimization procedure.

To proceed, we rewrite the constraints (4.8) as

$$
J_{i}(\mathbf{w}) \equiv \int_{\Omega} \mathrm{e}^{u_{0}^{i}+v_{i}} \mathrm{~d} x=K_{i}, \quad i=1, \ldots, m
$$

By the assumption (2.63), we have $K_{i}>0, i=1, \ldots, m$. Then we consider the constrained minimization problem

$$
\eta_{0} \equiv \inf \left\{I(\mathbf{w}) \mid \mathbf{w} \in W^{1,2}(\Omega) \text { and satisfies (4.23) }\right\} .
$$

If the problem (4.24) has a solution, say, $\tilde{\mathbf{w}}=\left(\tilde{w}_{1}, \ldots, \tilde{w}_{m}\right)$, then there exist some numbers (the Lagrangian multipliers) $\mu_{1}, \ldots, \mu_{m} \in \mathbb{R}$ such that

$$
\left(D\left(I+\mu_{1} J_{1}+\mu_{2} J_{2}+\cdots+\mu_{m} J_{m}\right)(\tilde{\mathbf{w}})\right)(\mathbf{w})=0, \quad \forall \mathbf{w} \in W^{1,2}(\Omega) .
$$

Using a series of test configurations $\mathbf{w}^{i}=\left(\delta_{i 1}, \ldots, \delta_{i m}\right), i=1, \ldots, m$ in (4.25) successively, we have

$$
\left.\begin{array}{rl}
L_{11} \mu_{1}+L_{21} \mu_{2} & =0 \\
L_{i i} \mu_{i}+L_{i+1 i} \mu_{i+1} & =0, \quad i=2, \ldots, m-1 \\
L_{m m} \mu_{m} & =0
\end{array}\right\}
$$

which imply $\mu_{1}=\mu_{2}=\cdots=\mu_{m}=0$. This is to say that, the constraints do not lead to the undesired Lagrangian multiplier problem, and any solution of the constrained minimization problem (4.25) is a critical point of the functional (4.18) itself.

From the constraint (4.23), we see that

$$
\begin{aligned}
& \underline{v}_{1}=L_{11} \underline{w}_{1}=\ln K_{1}-\ln \int_{\Omega} \mathrm{e}^{u_{0}^{1}+L_{11} \dot{w}_{1}} \mathrm{~d} x, \\
& \underline{v}_{i}=L_{i i-1} \underline{w}_{i-1}+L_{i i} \underline{w}_{i}=\ln K_{i}-\ln \int_{\Omega} \mathrm{e}^{u_{0}^{i}+L_{i i-1} \dot{w}_{i-1}+L_{i i} \dot{w}_{i}} \mathrm{~d} x, \quad i=2, \ldots, m .
\end{aligned}
$$

From (4.19), we have

$$
I(\mathbf{w})-\frac{1}{2 \lambda} \sum_{i=1}^{m}\left\|\nabla \dot{w}_{i}\right\|_{2}^{2}=\sum_{i=1}^{m} \int_{\Omega} \mathrm{e}^{\underline{v}_{i}} \mathrm{e}^{u_{0}^{i}+\dot{v}_{i}} \mathrm{~d} x-\sum_{i=1}^{m} \int_{\Omega} K_{i} \underline{v}_{i} \mathrm{~d} x,
$$

Plugging (4.28) into (4.29), and using Jensen's inequality, we have

$$
I(\mathbf{w})-\frac{1}{2 \lambda} \sum_{i=1}^{m}\left\|\nabla \dot{w}_{i}\right\|_{2}^{2} \geq-\sum_{i=1}^{m} K_{i} \ln K_{i}+\sum_{i=1}^{m} K_{i} \ln \sigma_{i}=\sum_{i=1}^{m} K_{i} \ln \frac{\sigma_{i}}{K_{i}},
$$

where $\sigma_{i}=|\Omega| \exp \left(\frac{1}{|\Omega|} \int_{\Omega} u_{0}^{i} \mathrm{~d} x\right), i=1, \ldots, m$. From (4.30) we know that the functional $I$ is bounded from below.

Let $\left\{\mathbf{w}^{(\ell)}\right\}=\left\{\left(w_{1}^{(\ell)}, \ldots, w_{m}^{(\ell)}\right)\right\}$ be a minimizing sequence of the problem (4.24). We conclude from (4.30) that $\left\{\dot{w}_{i}^{(\ell)}\right\}(i=1, \ldots m)$ are bounded in $W^{1,2}(\Omega)$. Without loss of generality, we may assume $\left\{\dot{w}_{i}^{(\ell)}\right\}(i=1, \ldots m)$ converge weakly in $W^{1,2}(\Omega)$. The Trudinger-Moser inequality 
(4.12) and (4.27) and (4.28) imply that $\left\{\underline{w}_{i}^{(\ell)}\right\}(i=1, \ldots m)$ also converge. Therefore, the sequence $\left\{\left(w_{1}^{(\ell)}, \ldots, w_{m}^{(\ell)}\right)\right\}$ has a weak limit in $W^{1,2}(\Omega)$ as $\ell \rightarrow \infty$. Noting that the constraint functionals are weakly continuous and the functional $I$ is weakly lower semi-continuous, we see that the weak limit of $\left\{\left(w_{1}^{(\ell)}, \ldots, w_{m}^{(\ell)}\right)\right\}$ must be a solution of the problem (4.24). Since $I$ is strictly convex, this solution is unique. Thus the constrained minimization procedure is carried out as well.

Finally, integrating the equations (4.2)-(4.4) or (4.5) over $\Omega$, we see that the quantized integrals (2.64) are established.

\section{The special case where $a=0$}

When $a=0$, we denote the matrix $R$ by $\dot{R}$. Then

$$
\dot{R}=\left(\begin{array}{cccccc}
1 & -1 & 0 & \ldots & \ldots & 0 \\
-1 & 3 & -2 & 0 & \ldots & 0 \\
0 & -2 & 5 & -3 & \ldots & 0 \\
\vdots & & \ddots & \ddots & \ddots & \vdots \\
0 & & \ddots & -(m-2) & 2 m-3 & -(m-1) \\
0 & \ldots & & 0 & -(m-1) & 2 m-1
\end{array}\right) .
$$

By a direct calculation, we see that the leading principal minors of $\dot{R}$ are

$$
\dot{R}_{i}=i !, \quad i=1, \ldots, m .
$$

Then by our formula (3.6) for the inverse of $\dot{R}$, we have

$$
\left(\dot{R}^{-1}\right)_{i j}=\sum_{l=j}^{m} \frac{1}{l}, \quad j=i, i+1, \ldots, m, \quad i=1, \ldots, m .
$$

that is

$$
\dot{R}^{-1}=\left(\begin{array}{ccccc}
\sum_{l=1}^{m} \frac{1}{l} & \sum_{l=2}^{m} \frac{1}{l} & \sum_{l=3}^{m} \frac{1}{l} & \ldots & \frac{1}{m} \\
\sum_{l=2}^{m} \frac{1}{l} & \sum_{l=2}^{m} \frac{1}{l} & \sum_{l=3}^{m} \frac{1}{l} & \ldots & \frac{1}{m} \\
\sum_{l=3}^{m} \frac{1}{l} & \sum_{l=3}^{m} \frac{1}{l} & \sum_{l=3}^{m} \frac{1}{l} & \ldots & \frac{1}{m} \\
\vdots & \vdots & \vdots & \ddots & \vdots \\
\frac{1}{m} & \frac{1}{m} & \frac{1}{m} & \ldots & \frac{1}{m}
\end{array}\right) .
$$

From this expression of $\dot{R}^{-1}$, we have

$$
r_{i} \equiv \sum_{j=1}^{m}\left(\dot{R}^{-1}\right)_{i j}=m-i+1, \quad i=1, \ldots, m .
$$

Thus, applying Theorem 2.3 , we obtain Theorem 2.4. 
An interesting feature of the case $a=0$ is that the matrix-valued charge densities $j^{0}$ and $J_{12}^{0}$ may coincide to satisfy the relation 33 .

$$
j^{0}=J_{12}^{0}=\frac{k}{2 \pi} B
$$

so that, using (2.48), the minimum energy is seen to be directly related to the vortex numbers according to the quantization formula

$$
\begin{aligned}
E & =\frac{1}{2} \mu\left|Q+2 R_{12}\right|=\frac{1}{3} \mu\left|\operatorname{Tr} \int j^{0} \mathrm{~d} x+2 \operatorname{Tr} \int J_{12}^{0} \mathrm{~d} x\right| \\
& =\frac{k \mu}{2 \pi}\left|\operatorname{Tr} \int B \mathrm{~d} x\right|=k \mu \sum_{i=1}^{N-1}(N-i) n_{i},
\end{aligned}
$$

as stated in 33 .

\section{Some concrete examples}

When $a>0$, the matrix computation quickly becomes rather involved for large $N$ and the results are not as explicit as the case for $a=0$. However, if $N$ is low and concrete, we can readily apply Theorem 2.3 to obtain explicit results for the problem. As an illustration, we work out the equations for $N=3$ (or $m=2$ ) and $N=4$ (or $m=3$ ), respectively, as examples.

We first consider the case when $m=2$. The equations in this case are

$$
\begin{aligned}
& \Delta u_{1}=\lambda\left(\left[2 a^{2}+1\right] \mathrm{e}^{u_{1}}-\left[a^{2}+1\right] \mathrm{e}^{u_{2}}-1\right)+4 \pi \sum_{s=1}^{n_{1}} \delta_{p_{1, s}}, \\
& \Delta u_{2}=\lambda\left(-\left[a^{2}+1\right] \mathrm{e}^{u_{1}}+\left[2 a^{2}+3\right] \mathrm{e}^{u_{2}}-1\right)+4 \pi \sum_{s=1}^{n_{2}} \delta_{p_{2, s}},
\end{aligned}
$$

for which the associated coefficient matrix $R$ reads

$$
R=\left(\begin{array}{cc}
2 a^{2}+1 & -\left(a^{2}+1\right) \\
-\left(a^{2}+1\right) & 2 a^{2}+3
\end{array}\right)
$$

with the eigenvalues

$$
\lambda_{1,2}=2 a^{2} \pm \sqrt{a^{4}+2 a^{2}+2}+2 .
$$

Therefore

$$
\lambda_{0}=2\left(2 a^{2}-\sqrt{a^{4}+2 a^{2}+2}+2\right) .
$$

On the other hand, the inverse of $R$ is

$$
R^{-1}=\frac{1}{3 a^{4}+6 a^{2}+2}\left(\begin{array}{cc}
2 a^{2}+3 & a^{2}+1 \\
a^{2}+1 & 2 a^{2}+1
\end{array}\right) .
$$

Thus, by definition, we have

$$
r_{1}=\frac{3 a^{2}+4}{3 a^{4}+6 a^{2}+2}, \quad r_{2}=\frac{3 a^{2}+2}{3 a^{4}+6 a^{2}+2} .
$$

In view of Theorem 2.3 , we obtain the following explicit results. 
Theorem 6.1 For any $a \geq 0, \lambda>0$, consider the equations (6.1)-(6.2).

(i) The equations (6.1)-(6.2) have a unique solution over $\mathbb{R}^{2}$ satisfying the boundary condition

$$
u_{1} \rightarrow \ln \frac{3 a^{2}+4}{3 a^{4}+6 a^{2}+2}, \quad u_{2} \rightarrow \ln \frac{3 a^{2}+2}{3 a^{4}+6 a^{2}+2}, \quad|x| \rightarrow \infty .
$$

Moreover, this solution obeys the following exponential decay estimate near infinity:

$$
\sum_{i=1}^{2}\left(u_{i}(x)-\ln r_{i}\right)^{2} \leq C(\varepsilon) \mathrm{e}^{-(1-\varepsilon) \sqrt{\lambda \lambda_{0}}|x|},
$$

where $\varepsilon \in(0,1)$ is arbitrarily small, $C(\varepsilon)$ is a positive constant depending on $\varepsilon, r_{1}, r_{2}$ are given by (6.7), and $\lambda_{0}$ is given by (6.5).

(ii) The equations (6.1)-(6.2) over a doubly periodic domain $\Omega$ have a solution if and only if the following two inequalities

$$
\begin{aligned}
& 4 \pi\left(\left[2 a^{2}+3\right] n_{1}+\left[a^{2}+1\right] n_{2}\right)<\lambda|\Omega|\left(3 a^{2}+4\right), \\
& 4 \pi\left(\left[a^{2}+1\right] n_{1}+\left[2 a^{2}+1\right] n_{2}\right)<\lambda|\Omega|\left(3 a^{2}+2\right),
\end{aligned}
$$

hold simultaneously. Furthermore, if a solution exists, it must be unique.

Next we consider the case when $m=3$. The equations are

$$
\begin{aligned}
& \Delta u_{1}=\lambda\left(\left[2 a^{2}+1\right] \mathrm{e}^{u_{1}}-\left[a^{2}+1\right] \mathrm{e}^{u_{2}}-1\right)+4 \pi \sum_{s=1}^{n_{1}} \delta_{p_{1, s}} \\
& \Delta u_{2}=\lambda\left(-\left[a^{2}+1\right] \mathrm{e}^{u_{1}}+\left[2 a^{2}+3\right] \mathrm{e}^{u_{2}}-\left[a^{2}+2\right] \mathrm{e}^{u_{3}}-1\right)+4 \pi \sum_{s=1}^{n_{2}} \delta_{p_{2, s}} . \\
& \Delta u_{3}=\lambda\left(-\left[a^{2}+2\right] \mathrm{e}^{u_{2}}+\left[2 a^{2}+5\right] \mathrm{e}^{u_{3}}-1\right)+4 \pi \sum_{s=1}^{n_{3}} \delta_{p_{3, s}},
\end{aligned}
$$

so that the associated coefficient matrix $R$ takes the form

$$
R=\left(\begin{array}{ccc}
2 a^{2}+1 & -\left(a^{2}+1\right) & 0 \\
-\left(a^{2}+1\right) & 2 a^{2}+3 & -\left(a^{2}+2\right) \\
0 & -\left(a^{2}+2\right) & 2 a^{2}+5
\end{array}\right)
$$

We will not compute all the eigenvalues of $R$. But, instead, we write down the inverse of $R$ :

$$
R^{-1}=\frac{1}{4 a^{6}+18 a^{4}+22 a^{2}+6}\left(\begin{array}{ccc}
3 a^{4}+12 a^{2}+11 & 2 a^{4}+7 a^{2}+5 & a^{4}+3 a^{2}+2 \\
2 a^{4}+7 a^{2}+5 & 4 a^{4}+12 a^{2}+5 & 2 a^{4}+5 a^{2}+2 \\
a^{4}+3 a^{2}+2 & 2 a^{4}+5 a^{2}+2 & 3 a^{4}+6 a^{2}+2
\end{array}\right),
$$

which gives us

$$
\begin{aligned}
& r_{1}=\frac{3 a^{4}+11 a^{2}+9}{2 a^{6}+9 a^{4}+11 a^{2}+3}, \\
& r_{2}=\frac{4 a^{4}+12 a^{2}+6}{2 a^{6}+9 a^{4}+11 a^{2}+3}, \\
& r_{3}=\frac{3 a^{4}+7 a^{2}+3}{2 a^{6}+9 a^{4}+11 a^{2}+3} .
\end{aligned}
$$

Applying Theorem 2.3 again, we can state 
Theorem 6.2 For any $a \geq 0, \lambda>0$, consider the equations (6.12)-(6.14).

(i) The equations have a unique solution over $\mathbb{R}^{2}$ satisfying the boundary condition

$$
u_{i} \rightarrow \ln r_{i}, \quad|x| \rightarrow \infty, \quad i=1,2,3,
$$

where $r_{1}, r_{2}, r_{3}$ are defined by (6.17) -(6.19). Moreover, this solution satisfies the following exponential decay estimate at infinity:

$$
\sum_{i=1}^{3}\left(u_{i}(x)-\ln r_{i}\right)^{2} \leq C(\varepsilon) \mathrm{e}^{-(1-\varepsilon) \sqrt{\lambda \lambda_{0}}|x|},
$$

where $\varepsilon \in(0,1)$ is small, $C(\varepsilon)>0$ depends on $\varepsilon$, and $\lambda_{0}$ is twice the smallest eigenvalue of $R$.

(ii) The equations (6.12) -(6.14) over a doubly periodic domain $\Omega$ have a solution if and only if the following three inequalities

$$
\begin{aligned}
2 \pi\left(\left[3 a^{4}+12 a^{2}+11\right] n_{1}+\left[2 a^{4}+7 a^{2}+5\right] n_{2}+\left[a^{4}+3 a^{2}+2\right] n_{3}\right) & <\lambda|\Omega|\left(3 a^{4}+11 a^{2}+9\right), \\
\pi\left(\left[2 a^{4}+7 a^{2}+5\right] n_{1}+\left[4 a^{4}+12 a^{2}+5\right] n_{2}+\left[2 a^{4}+5 a^{2}+2\right] n_{3}\right) & <\lambda|\Omega|\left(2 a^{4}+6 a^{2}+3\right), \\
2 \pi\left(\left[1 a^{4}+3 a^{2}+2\right] n_{1}+\left[2 a^{4}+5 a^{2}+2\right] n_{2}+\left[3 a^{4}+6 a^{2}+2\right] n_{3}\right) & <\lambda|\Omega|\left(3 a^{4}+7 a^{2}+3\right),
\end{aligned}
$$

hold simultaneously. Furthermore, if a solution exists, it must be unique.

In general, for any $N \geq 2$, since we have established the existence and uniqueness of a solution realizing $n_{i}$ arbitrarily prescribed zeros for each $f_{i}, i=1, \ldots, N-1$, we see that the solutions precisely depend on

$$
2 n \equiv 2 \sum_{i=1}^{N-1} n_{i}
$$

continuous parameters which are the coordinates of the vortex points

$$
p_{1,1}, \ldots, p_{1, n_{1}}, \ldots, p_{N-1,1}, \ldots, p_{N-1, n_{N-1}} .
$$

It may be relevant to recall the index-theory study of Weinberg [49] on the Abelian Higgs BPS equations which shows that the dimension of the moduli space of multiple vortex solutions is $2 n$. This number is precisely the number of the coordinates in $\mathbb{R}^{2}$ which are needed to determine the locations of $n$ vortices, in view of the classical existence and uniqueness results in [31]. There are similar studies in the context of non-relativistic Chern-Simons equations 34] and of superconducting cosmic strings [42. It will be interesting to know the dimension of the moduli space of the multiple vortex solutions of the BPS vortex equations (2.15)-(2.17) of Kim-Kim-Kwon-Nakajima [33] in the ABJM theory. Thus our existence and uniqueness results indicate that, within the ansatz of Kim-Kim-Kwon-Nakajima [33], the dimension of the moduli space of the $n$-vortex solutions of the BPS equations (2.15)-(2.17) is also $2 n$, although, generally when no specific ansatz is specified, it should depend on the gauge group index $N$ as well [17].

\section{References}

[1] A. A. Abrikosov, On the magnetic properties of superconductors of the second group, Sov. Phys. JETP 5 (1957) 1174-1182. 
[2] O. Aharony, O. Bergman, D. L. Jaferis and J. Maldacena, $\mathcal{N}=6$ superconformal ChernSimons-matter theories, M2-branes and their gravity duals, J. High Energy Phys. 0810 (2008) 091.

[3] T. Aubin, Nonlinear Analysis on Manifolds: Monge-Ampére Equations, Springer, Berlin and New York, 1982.

[4] R. Auzzi and S. P. Kumar, Non-Abelian vortices at weak and strong coupling in mass deformed ABJM theory. J. High Energy Phys. 0710910 (2009).

[5] J. Bagger and N. Lambert, Modeling multiple M2's, Phys. Rev. D 75 (2007) 045020.

[6] J. Bagger and N. Lambert, Gauge symmetry and supersymmetry of multiple M2-branes, Phys. Rev. D 77 (2008) 065008.

[7] J. Bagger and N. Lambert, Comments on multiple M2-branes, J. High Energy Phys. 0802 (2008) 105.

[8] M. A. Bandres, A. E. Lipstein, and J. H. Schwarz, Studies of the ABJM theory in a formulation with manifest SU(4) R-symmetry, J. High Energy Phys. 0809 (2008) 027.

[9] D. Belyaev, L. Brink, S.-S. Kim, and P. Ramond, The BLG theory in light-cone superspace, J. High Energy Phys. 1004 (2010) 026.

[10] E. B. Bogomol'nyi, The stability of classical solutions, Sov. J. Nucl. Phys. 24 (1976) 449-454.

[11] L. Caffarelli and Y. Yang, Vortex condensation in the Chern-Simons Higgs model: an existence theorem, Commun. Math. Phys. 168 (1995) 321-336.

[12] D. Chae and O. Yu. Imanuvilov, The existence of nontopological multivortex solutions in the relativistic self-dual Chern-Simons theory, Commun. Math. Phys. 215 (2000) 119-142.

[13] S. Chakrabortty, S. P. Chowdhury, and K. Ray, Some BPS configurations of the BLG theory, Phys. Lett. B 703 (2011) 172-179.

[14] H. Chan, C. C. Fu, and C. S. Lin, Non-topological multivortex solutions to the self-dual Chern-Simons-Higgs equation, Commun. Math. Phys. 231 (2002) 189-221.

[15] S. Chen and Y. Yang, Existence of multiple vortices in supersymmetric gauge field theory, Proc. R. Soc. A, in press.

[16] G. Dunne, Self-Dual Chern-Simons Theories, Lecture Notes in Physics, vol. m 36, Springer, Berlin, 1995.

[17] M. Eto, Y. Isozumi, M. Nitta, K. Ohashi, and N. Sakai, Moduli space of non-Abelian vortices, Phys. Rev. Lett. 96 (2006) 161601.

[18] B. Ezhuthachan, S. Mukhi, and C. Papageorgakis, The power of the Higgs mechanism: higherderivative BLG theories, J. High Energy Phys. 0904 (2009) 101. 
[19] L. Fontana, Sharp borderline Sobolev inequalities on compact Riemannian manifolds, Comment. Math. Helv. 68 (1993) 415-454.

[20] J. Fröhlich, The fractional quantum Hall effect, Chern-Simons theory, and integral lattices, Proc. Internat. Congr. Math., pp. 75-105, Birkhäuser, Basel, 1995.

[21] J. Fröhlich and P. Marchetti, Quantum field theory of anyons, Lett. Math. Phys. 16 (1988) $347-358$.

[22] J. Fröhlich and P. Marchetti, Quantum field theory of vortices and anyons, Commun. Math. Phys. 121 (1989) 177-223.

[23] G. H. Golub and J. M. Ortega, Scientific Computing and Differential Equations, Academic, San Diego, 1992.

[24] A. Gustafson, I. M. Sigal, and T. Tzaneteas, Statics and dynamics of magnetic vortices and of Nielsen-Olesen (Nambu) strings, J. Math. Phys. 51 (2010) 015217.

[25] A. Gustavsson, Algebraic structures on parallel M2-branes, Nucl. Phys. B 811 (2009) 66-76.

[26] G. 't Hooft, A property of electric and magnetic flux in non-Abelian gauge theories, Nucl. Phys. B 153 (1979) 141-160.

[27] K. Hosomichi, K. Lee, and S. Lee, Mass-deformed Bagger-Lambert theory and its BPS objects, Phys. Rev. D 78 (2008) 066015.

[28] J. Hong, Y. Kim and P.-Y. Pac, Multivortex solutions of the Abelian Chern-Simons-Higgs theory, Phys. Rev. Lett. 64 (1990) 2330-2333.

[29] R. Jackiw, S.-Y. Pi, and E. J. Weinberg, Topological and non-topological solitons in relativistic and non-relativistic Chern-Simons theory, Particles, Strings and Cosmology (Boston, 1990), pp. 573-588, World Sci. Pub., River Edge, NJ, 1991.

[30] R. Jackiw and E. J. Weinberg, Self-dual Chern-Simons vortices, Phys. Rev. Lett. 64 (1990) $2334-2337$.

[31] A. Jaffe and C. H. Taubes, Vortices and Monopoles, Birkhäuser, Boston, 1980.

[32] C. Kim, Vortex-type solutions in ABJM theory, Journal of Physics: Conference Series 343 (2012) 012057.

[33] C. Kim, Y. Kim, O. K. Kwon, and H. Nakajima, Vortex-type half-BPS solitons in ABJM theory, Phys. Rev. D 80 (2009) 045013.

[34] S. K. Kim, K. S. Soh, and J. H. Yee, Index theory for the nonrelativistic Chern-Simons solitons Phys. Rev. D 42 (1990) 4139-4144.

[35] E. H. Lieb and Y. Yang, Non-Abelian vortices in supersymmetric gauge field theory via direct methods, Commun. Math. Phys. 313 (2012) 445-478 
[36] C. S. Lin and Y. Yang, Non-Abelian multiple vortices in supersymmetric field theory, Comm. Math. Phys. 304 (2011) 433-457,

[37] C. S. Lin and Y. Yang, Sharp existence and uniqueness theorems for non-Abelian multiple vortex solutions, Nucl. Phys. B 846 (2011) 650-676.

[38] H. Nielsen and P. Olesen, Vortex-line models for dual strings, Nucl. Phys. B 61 (1973) 45-61.

[39] M. Nolasco and G. Tarantello, Vortex condensates for the $S U(3)$ Chern-Simons theory, Commun. Math. Phys. 213 (2000) 599-639.

[40] M. K. Prasad and C. M. Sommerfield, Exact classical solutions for the 't Hooft monopole and the Julia-Zee dyon, Phys. Rev. Lett. 35 (1975) 760-762.

[41] J. H. Schwarz, Superconformal Chern-Simons theories, J. High Energy Phys. 0411 (2004) 078.

[42] G. W. Semenoff, Index theorems and superconducting cosmic strings, Phys. Rev. D 37 (1988) $2838-2852$.

[43] J. Spruck and Y. Yang, Topological solutions in the self-dual Chern-Simons theory: existence and approximation, Ann. Inst. H. Poincaré - Anal. non Linéaire 12 (1995) 75-97.

[44] J. Spruck and Y. Yang, The existence of non-topological solitons in the self-dual Chern-Simons theory, Commun. Math. Phys. 149 (1992) 361-376.

[45] G. Tarantello, Multiple condensate solutions for the Chern-Simons-Higgs theory, J. Math. Phys. 37 (1996) 3769-3796.

[46] G. Tarantello, Self-Dual Gauge Field Vortices, Progress in Nonlinear Differential Equations and Their Applications 72, Birkhäuser, Boston, 2008.

[47] C. H. Taubes, Arbitrary $N$-vortex solutions to the first order Ginzburg-Landau equations, Commun. Math. Phys. 72 (1980) 277-292.

[48] S. Wang and Y. Yang, Abrikosov's vortices in the critical coupling, SIAM J. Math. Anal. 23 (1992) 1125-1140.

[49] E. J. Weinberg, Multivortex solutions of the Ginzburg-Landau equations, Phys. Rev. D 19 (1979) 3008-3012.

[50] F. Wilczek, Fractional Statistics and Anyonic Superconductivity, World Scientific, Singapore, 1990.

[51] Y. Yang, The relativistic non-Abelian Chern-Simons equations, Commun. Math. Phys. 186 (1997) 199-218.

[52] Y. Yang, On a system of nonlinear elliptic equations arising in theoretical physics, J. Funct. Anal. 170 (2000) 1-36.

[53] Y. Yang, Solitons in Field Theory and Nonlinear Analysis, Springer, New York, 2001. 